\title{
On the role of kinetic energy during unstable propagation in a heterogeneous peeling test
}

\author{
Giuliano Lazzaroni • Renaud Bargellini - Pierre-Emmanuel Dumouchel • \\ Jean-Jacques Marigo
}

December 1, 2011

\begin{abstract}
We study the dynamic debonding of a onedimensional inextensible film, subject to a monotonic loading and under the hypothesis that the toughness of the glue can take only two values. We first consider the case of a single defect of small length in the glue where the toughness is lower than in the remaining part. The dynamic solution is obtained in a closed form and we prove that it does not converge to the expected quasistatic one when the loading speed tends to zero. The gap is due to a kinetic energy which appears when the debonding propagates across the defect at a velocity which is of the same order as the sound velocity. The kinetic energy becomes negligible again only when the debonding has reached a critical distance beyond the defect. The case of many defects is then considered and solved using an exact numerical solution of the wave equation and the Griffith law of propagation. The numerical results highlight the effects of the time evolution of the kinetic energy which induce alternate phases of
\end{abstract}

G. Lazzaroni (Corresponding author)

UPMC and CNRS, UMR 7190, Institut Jean Le Rond d'Alembert, F-75005 Paris, France

and

Universität Würzburg, Institut für Mathematik, EmilFischer-Straße 40, 97074 Würzburg, Germany

R. Bargellini

EDF R\&D, LAboratoire de Mécanique des Structures Industrielles Durables, UMR CNRS/EDF 2832, 1 Avenue du Général De Gaulle, 92141 Clamart Cedex, France

P.-E. Dumouchel

Peugeot-Citroen Automobile, Département Boîtes de Vitesses, 92256 La Garenne-Colombes, France

J.-J. Marigo

École Polytechnique, Laboratoire de Mécanique des Solides, 91128 Palaiseau Cedex, France

E-mail: giuliano.lazzaroni@uni-wuerzburg.de (G. Lazzaroni) rapid and slow debonding, these oscillations depending essentially on the volume fraction of the highest toughness.

Keywords brittle fracture - crack propagation · Griffith's criterion · dynamic fracture · quasistatic evolution · shock waves · nonlinear stability

PACS 62.20.mj - 62.50.Ef - 43.25.Cb - 68.35.Ja · 83.60.Uv

\section{Introduction}

This paper is a contribution to the study of dynamic fracture in the framework of Griffith's theory [4,11,12, 17]. We are interested in analyzing the propagation of a crack in a heterogeneous material (with variable toughness), understanding the role of the kinetic energy. In particular, we wonder whether a (possibly modified) quasistatic model is a good approximation of the phenomenon, avoiding delicate and time-consuming dynamic computations.

Indeed, even under quasistatic loading (i.e., assuming that the speed of loading is smaller than the speed of the internal vibrations), the material's answer is a priori dynamic, because Griffith's theory does not provide an absolutely continuous solution when the crack passes through a zone where the toughness suddenly decreases $[9,2,18]$. In this case, since the energy release rate becomes greater than the toughness, the dynamic evolution presents a fast propagation, whose speed has the order of the speed of sound; in a quasistatic framework, the counterparts of fast propagations are jumps in time.

Hence, one deals with a free-boundary problem of elastodynamics, driven by Griffith's dynamic law. In order to capture the nontrivial behaviour of the kinetic 
energy, we consider here the simplified model like in $[16,6,7]$ : we study the debonding of a one-dimensional inextensible film, subject to a monotonic loading with vanishing speed, under the hypothesis that the toughness is piecewise constant and takes only two possible values.

It is quite surprising to see that, at least at our knowledge, this "elementary" problem was never solved in the literature. Indeed, even if there exists a great number of papers devoted to the case of debonding in the homogeneous case, see for instance [10,13-15], very few consider the heterogeneous case. One can find works like $[5,22]$ devoted to dynamic delamination of Double Cantilever Beams where the heterogeneities are thoughthickness reinforcements. However, the heterogeneities are there treated as continuously distributed bridging cohesive zones and this modelling leads to a different type of effective dynamic behaviour. In particular, it cannot capture the transitory effects of kinetic energy due to the back and forth of shock waves created when the crack tip crosses the interfaces.

Here, we follow the approach of $[6,7]$, where a complete analysis is performed in the case of a single discontinuity of the toughness (increasing or decreasing). In particular, in the case of toughness with one discontinuity, the results of [7] show that the dynamic solutions converge, as the loading speed tends to zero, towards the quasistatic evolution, upon assuming a criterion of energy conservation to govern the jumps. So the quasistatic approximation is good, even if the kinetic energy reaches a large value during the fast propagation. Moreover, it is possible to determine the length of the crack's jump and the time occurring for the debonding to restart after the jump.

In this work, we consider the case of a defect, i.e., the case of two discontinuities of the toughness. If the size of the defect does not exceed a threshold depending only on the two possible values of the toughness, we prove that the dynamic solutions do not converge to the usual quasistatic one: indeed, the jump's length is overestimated by the usual quasistatic evolution (see Proposition 6 and Figure 6). Hence, the kinetic energy cannot be neglected, so we provide an "optimal bound" on it (Proposition 9): it turns out that the kinetic energy increases during the fast propagation and is totally relaxed when the system comes back to a quasistatic behaviour. Nevertheless, a good approximation is represented by a quasistatic model where the toughness is suitably modified: we give the expression of the alternative toughness in Proposition 7.

Finally, we consider the case of many defects, whose number diverges and whose distribution could be periodic or random. In the quasistatic model (under the assumption of energy conservation), the solutions are staircase functions in the space/time plane, bounded by lines whose slope depends on the density of material with the lowest toughness. In particular, fixed a level of load, the length of debonding can be higher than the one corresponding to a material with a constant low toughness. Moreover, even if the density of the lowest toughness tends to one, in the limit the behaviour is different from the case of a homogeneous material.

Due to the difficulty of controlling the several interactions that perturb the debonding process, in the case of many defects the dynamic problem is treated using an exact numerical solution of the wave equation. The simulations show that the qualitative aspect of the dynamic solutions depends strongly on the density of the lowest toughness, while it is not affected by the choice of the defects' distribution.

If the density of the lowest toughness is close to one, the dynamic solutions seem to converge to a quasistatic staircase evolution: indeed, during the fast propagations the debonding passes through many defects. As this density decreases, the curves in the space/time plane seem smoother, showing a wave trend; if the density is close to $1 / 2$, their shape approaches a line, whose slope is however different from the one corresponding to the mean toughness.

This behaviour reflects the influence of the kinetic energy on the dynamics. Indeed, we see here the competition of two opposite phenomena: the creation of kinetic energy as the toughness decreases and the relaxation observed in the case of a single defect.

This paper is organized as follows. In Section 1 we introduce the main ingredients of the model and we summarize the results in the case of a single discontinuity of the toughness. Section 2 contains the analysis of the case of a defect (two discontinuities), both in the quasistatic and the dynamic framework. Since the dynamics does not tend to the quasistatics, we show in Section 2.4 the possible modification to the toughness in order to ensure convergence. Finally, we study the kinetic energy in Section 2.5. In Section 3 we discuss the numerical results for the case of many defects.

\section{Convergence to a discontinuous quasistatic solution}

We introduce the problem of a simplified peeling test for a thin film, comparing quasistatics and dynamics. In [7] it is presented an example where the dynamic solutions converge, as the speed of loading tends to zero, to a discontinuous quasistatic evolution. We summarise here the main features of the model, referring to [7] for further details. 
Fig. 1 Simplified peeling test.

\subsection{Simplified peeling test}

We consider a simplified version of the peeling test $[17$, 21]. A semi-infinite, perfectly flexible, and inextensible thin film is initially bonded to a rigid substrate with normal $\mathbf{e}_{2}$ (Figure 1). Its end $x_{1}=0$ is submitted both to a constant tension $-N \mathbf{e}_{1}$, with $N>0$, and to an opening displacement $W$; as $W$ increases, the front of debonding propagates. The film has a toughness $G_{c}\left(x_{1}\right)$, depending only on $x_{1}$, which is assumed to be piecewise constant.

In order to shorten the expressions, we introduce dimensionless quantities. For this purpose, we consider a specific characteristic length $L$ of the film and we set $x:=\frac{x_{1}}{L}, \quad \mathrm{~T}:=\frac{\mathrm{W}}{\mathrm{L}}$.

The displacement field $\mathbf{u}$ of the film can then be expressed as a function of $(x, T)$ defined on $\mathcal{Q}:=(0,+\infty)^{2}$,

$\mathbf{u}(x, T)=u(x, T) L \mathbf{e}_{\mathbf{1}}+w(x, T) L \mathbf{e}_{\mathbf{2}}$.

If $w$ is small enough, using a linearized inextensibility condition we can express $u$ in terms of $w$,

$u(x, T)=\frac{1}{2} \int_{x}^{\infty}\left(\frac{\partial w}{\partial x}(s, T)\right)^{2} \mathrm{~d} s$,

so the only unknown of the problem is $w$.

The debonded part of the film for a given loading $T$ corresponds to the points $x$ such that $\sup _{S \leqslant T} w(x, S)>$ 0 . The debonding is assumed to grow from $x=0$ at $T=0$ in such a way that the debonded part of the film corresponds to an interval $(0, \ell(T)$ ) (notice that $\ell(T) L$ represents the physical debonded length). Therefore, we have

$u=w=0 \quad$ in $\mathcal{Q}_{0}:=\left\{(x, T) \in(0,+\infty)^{2}: x \geqslant \ell(T)\right\}$.

In our analysis, the various energies play a major role. Since the film is perfectly flexible, the potential energy is equal to the opposite of the work produced by the tension $N$ in the displacement $u(0, T)$; by (3), it reads

$\mathcal{P}(T):=\frac{N L}{2} \int_{0}^{\infty}\left(\frac{\partial w}{\partial x}(x, T)\right)^{2} \mathrm{~d} x$.
The debonding of the film is assumed to be governed by Griffith's law [12]. The surface energy of the film is then given by

$\mathcal{S}(T):=L \int_{0}^{\ell(T} \mathcal{G}_{c}(x)\left(x_{x}\right)$.

In the following sections we present the main tools for the quasistatic and the dynamic analysis.

\subsection{Ingredients for the quasistatic analysis}

In the quasistatic approach, the film is assumed to be in equilibrium for every value of the given loading $T$. Minimizing the potential energy (5) over the functions $w$ such that $w \geqslant 0, w(0)=T$, and $w=0$ in $[\ell,+\infty)$ (where $\ell>0$ is a fixed length), one sees [7, Section 2.2] that the transverse displacement field at equilibrium is

$$
w^{0}(x):=T\left(1-\frac{x}{\ell}\right)^{+},
$$

where $(\cdot)^{+}$stands for the positive part. The corresponding energies are

$$
\mathcal{P}(T, \ell)=\frac{N L T^{2}}{2 \ell}, \quad \mathcal{S}(\ell)=\mathrm{L} \int_{0}^{\ell} \mathrm{G}_{\mathrm{c}}(\mathrm{x}) \mathrm{dx} .
$$

The quasistatic potential energy release rate, which is defined as the opposite of the derivative of the potential energy with respect to $\ell$, consequently reads

$G(T, \ell):=-\frac{1}{L} \frac{\partial \mathcal{P}}{\partial \ell}(T, \ell)=\frac{N T^{2}}{2 \ell^{2}}$.

The quasistatic total energy $\mathcal{E}^{q}(T, \ell)$ is given by

$\mathcal{E}^{q}(T, \ell):=\mathcal{P}(T, \ell)+\mathcal{S}(\ell)=\frac{N L T^{2}}{2 \ell}+L \int_{0}^{\ell} G_{c}(x) \mathrm{d} x$.

Given $T>0$, the function $\ell \mapsto \mathcal{E}^{q}(T, \ell)$ is strictly convex if and only if $x \mapsto G_{c}(x)$ is nondecreasing.

The quasistatic evolution of the length of debonding $\ell(T)$ is a function of the loading $T$, satisfying Griffith's criterion: $T \mapsto \ell(T)$ must be absolutely continuous (see, e.g., [8] for the definition of absolutely continuous function) and must satisfy for a.e. $T>0$

$\left\{\begin{array}{l}\dot{\ell}(T) \geqslant 0, \\ \frac{N T^{2}}{2 \ell^{2}(T)} \leqslant G_{c}(\ell(T)), \\ \left(\frac{N T^{2}}{2 \ell^{2}(T)}-G_{c}(\ell(T))\right) \dot{\ell}(T)=0,\end{array}\right.$

with the initial condition $\ell(0)=0$. In (9) the first inequality is the irreversibility condition, the second is 
Griffith's criterion (the energy release rat $\underset{\text { fis }}{\text { is always }}$ replacements bounded by the local toughness $\left.G_{c}(\ell)\right)$, and the last $\ell_{1}$ equality prescribes that the debonding evolves only when $\ell_{c}$ the energy release rate is critical.

We recall that by assumption the toughness of the material is a piecewise constant function of $x_{1}$. During the first phase (before the first change of toughness), the evolution of the debonding $T \mapsto \ell(T)$ can be determined by Griffith's law (9). However, after the change of toughness, the results of [7] show that there exists a unique absolutely continuous solution in the case of a toughness increase, whereas there is no absolutely continuous solution in the case of a toughness decrease.

More precisely, let us consider the two cases:

- Case (a) (increasing toughness):

$$
G_{c}(x)= \begin{cases}N \gamma_{1} & \text { if } 0<x \leqslant \ell_{1} \\ N \gamma_{2} & \text { if } x>\ell_{1}\end{cases}
$$

- Case (b) (decreasing toughness):

$$
G_{c}(x)= \begin{cases}N \gamma_{2} & \text { if } 0<x \leqslant \ell_{1} \\ N \gamma_{1} & \text { if } x>\ell_{1}\end{cases}
$$

where $\ell_{1}>0$ and $0<\gamma_{1}<\gamma_{2}$ in both cases.

In case (a), the debonding evolves with speed $1 / \sqrt{2 \gamma_{1}}$ for $T \leqslant T_{1}:=\ell_{1} \sqrt{2 \gamma_{1}}$. Then by (9) it stops at $\ell_{1}$, since $G\left(T_{1}, \ell_{1}\right)=G_{c}\left(\ell_{1}^{-}\right)<G_{c}\left(\ell_{1}^{+}\right)$. The debonding restarts when the loading $T$ is so large that the energy release rate $G\left(T, \ell_{1}\right)$ equals again $G_{c}\left(\ell_{1}^{+}\right)$, namely when $T=\widetilde{T}_{1}:=\ell_{1} \sqrt{2 \gamma_{2}}$, and propagates with speed $1 / \sqrt{2 \gamma_{2}}$ (see Figure 2).

On the other hand, in case (b) the debonding evolution is necessarily discontinuous: $\ell$ must jump from $\ell_{1}$ (the discontinuity point) to some point $\ell_{c}>1$, impossible to determine using Griffith's law. Therefore, in [7] the problem is set in an extended quasistatic framework, under a principle of energy conservation (see also $[20,19,2]$ for a general variational approach to rate independent behaviours): this permits one to deal with the unstable crack propagation phase. More precisely, at $T_{1}:=\ell_{1} \sqrt{2 \gamma_{2}}$ the debonding jumps from $\ell_{1}$ to a value $\ell_{c}:=\ell_{1} \frac{\gamma_{2}}{\gamma_{1}}$ such that $\mathcal{E}^{q}\left(T_{1}, \ell_{1}\right)=\mathcal{E}^{q}\left(T_{1}, \ell_{c}\right)$. Afterwards, since the energy release rate is less than the toughness, the debonding stops and its length remains constant at the value $\ell_{c}$ : this holds as long as $T \leqslant \widetilde{T}_{c}:=2 \gamma_{2} / \sqrt{2 \gamma_{1}}$, where the energy release rate is again critical. Then the debonding restarts with speed $1 / \sqrt{2 \gamma_{1}}$ (see Figure 2).

The criterion of conservation of the total quasistatic energy thus provides a solution for the unstable crack propagation, i.e., when Griffith's law fails. In addition, in both cases the solution coincides with the limit of some dynamic solutions as the loading speed tends to zero, as we will see in the next section.

$\ell$
$T$
$T_{1}$
$\widetilde{T}_{1}$
$T_{1}$
$\widetilde{T}_{c}$

Fig. 2 Quasistatic evolution with a single discontinuity of the toughness: left, case (a); right, case (b).

\subsection{Ingredients for the dynamic analysis}

Following [7, Section 2.1], we present the tools to analyse the dynamics of the peeling test, in order to treat the case of unstable (discontinuous) crack propagation. The loading is assumed to have a dimensionless speed $\varepsilon>0$,

$W=\varepsilon c t$

where $c:=\sqrt{\frac{N}{\rho}}$ is the velocity of the transversal waves propagating in the film and $\rho$ is the mass of a unit length of the film. For the whole paper the speed $\varepsilon$ will be assumed small enough.

As above, we denote by $\ell^{\varepsilon}(T)$ the length of the debonded part and by $w^{\varepsilon}$ the transverse component of the displacement field (see (2)). Its partial derivatives are

$\omega^{\varepsilon}:=\frac{\partial w^{\varepsilon}}{\partial x}, \quad \mathrm{v}^{\varepsilon}:=\frac{\partial \mathrm{w}^{\varepsilon}}{\partial \mathrm{T}}$,

where the rescaled variables $(x, T)$ are given in (1). The field $\omega^{\varepsilon}$ represents the infinitesimal rotation of the film, while the field $v^{\varepsilon}$ is the rescaled transverse velocity (the actual transverse velocity of material points being $\left.\varepsilon c v^{\varepsilon}\right)$.

The potential and the surface energy are defined by

$\mathcal{P}^{\varepsilon}(T):=\frac{N L}{2} \int_{0}^{\infty} \omega^{\varepsilon}(x, T)^{2} \mathrm{~d} x$

and

$\mathcal{S}^{\varepsilon}(T):=L \int_{0}^{\ell^{\varepsilon}(T)} G_{c}(x) \mathrm{d} x$,

respectively. The kinetic energy of the film at time $t$ is defined as

$\mathcal{K}^{\varepsilon}(T):=\varepsilon^{2} \frac{N L}{2} \int_{0}^{\infty} v^{\varepsilon}(x, T)^{2} \mathrm{~d} x$,

where the factor $\varepsilon^{2}$ is due to the rescaling of time. 
The debonded part of the film is governed by the equation of motion [1]

$\frac{\partial \omega^{\varepsilon}}{\partial x}-\varepsilon^{2} \frac{\partial v^{\varepsilon}}{\partial T}=0 \quad$ in $\mathcal{Q} \backslash\left(\mathcal{Q}_{0}^{\varepsilon} \cup S^{\varepsilon}\right)$,

where $\mathcal{Q}_{0}^{\varepsilon}:=\left\{(x, T) \in(0,+\infty)^{2}: x \geqslant \ell^{\varepsilon}(T)\right\}$ and $S^{\varepsilon}$ is the set of the discontinuity points of $\omega^{\varepsilon}$ and $v^{\varepsilon}$ in $\mathcal{Q} \backslash \mathcal{Q}_{0}^{\varepsilon}$ (see also the definition below). In addition, the Hadamard compatibility condition gives

$\frac{\partial \omega^{\varepsilon}}{\partial T}-\frac{\partial v^{\varepsilon}}{\partial x}=0 \quad$ in $\mathcal{Q} \backslash\left(\mathcal{Q}_{0}^{\varepsilon} \cup S^{\varepsilon}\right)$.

Notice that (17) and (18) are equivalent to the wave equation [1]

$\frac{\partial^{2} w^{\varepsilon}}{\partial x^{2}}-\varepsilon^{2} \frac{\partial^{2} w^{\varepsilon}}{\partial T^{2}}=0 \quad$ in $\mathcal{Q} \backslash\left(\mathcal{Q}_{0}^{\varepsilon} \cup S^{\varepsilon}\right)$.

Finally, the initial condition (4) holds on $\mathcal{Q}_{0}^{\varepsilon}$. Moreover, due to the rescaling $v^{\varepsilon}$ satisfies at $x=0$ the boundary condition

$v^{\varepsilon}(0, T)=1$

for every $T>0$.

As shown in [7], the change of toughness at $\ell_{1}$ generates a backward shock wave $S_{1}^{\varepsilon-}$, i.e., a discontinuity point for $\omega^{\varepsilon}$ and $v^{\varepsilon}$; moreover, at $\ell_{1}$ the debonding speed changes. The backward shock wave is then reflected on the axis $x=0$ and transformed into a forward shock wave $S_{1}^{\varepsilon+}$, which intersects the front of debonding modifying again the speed. At the intersection, the forward shock wave is replaced by a backward shock wave $S_{2}^{\varepsilon-}$, and so on. The backward shock waves, denoted by $S^{\varepsilon-}:=\bigcup_{m} S_{m}^{\varepsilon-}$, propagate with a local velocity $s=-1 / \varepsilon$, while the forward shock waves, denoted by $S^{\varepsilon+}:=\bigcup_{m} S_{m}^{\varepsilon+}$, propagate with velocity $s=1 / \varepsilon$. On $S^{\varepsilon}:=S^{\varepsilon-} \cup S^{\varepsilon+}$, equations (17)-(18) are replaced by the Hadamard condition and the Rankine-Hugoniot relation [23],

$\llbracket \omega^{\varepsilon} \rrbracket+\varepsilon \llbracket v^{\varepsilon} \rrbracket=0 \quad$ on $S^{\varepsilon+}, \quad \llbracket \omega^{\varepsilon} \rrbracket-\varepsilon \llbracket \mathrm{v}^{\varepsilon} \rrbracket=0 \quad$ on $\mathrm{S}^{\varepsilon-}$.

In the last equations, $\llbracket f \rrbracket$ stands for the jump of $f$ across $S^{\varepsilon}$, i.e., $\llbracket f \rrbracket:=f_{+}-f_{-}$on $S^{\varepsilon}, f_{+}$and $f_{-}$denoting respectively the limit after and before the passage of the shock wave.

We now present the law to determine the evolution of the front of debonding $\Gamma^{\varepsilon}:=\left\{\left(\ell^{\varepsilon}(T), T\right): T>0\right\}$. On $\Gamma^{\varepsilon}$ the displacement $w^{\varepsilon}$ is continuous (and equal to 0 ), but $\omega^{\varepsilon}$ and $v^{\varepsilon}$ are discontinous and satisfy the compatibility condition

$\llbracket v^{\varepsilon} \rrbracket+\dot{\ell}^{\varepsilon} \llbracket \omega^{\varepsilon} \rrbracket=v_{+}^{\varepsilon}+\dot{\ell}^{\varepsilon} \omega_{+}^{\varepsilon}=0 \quad$ on $\Gamma^{\varepsilon}$.
In the last equation, we have used (4) and we have denoted by $\llbracket f \rrbracket$ the difference $f_{+}-f_{-}$between the limit in $\mathcal{Q} \backslash \mathcal{Q}_{0}^{\varepsilon}$ and in $\mathcal{Q}_{0}^{\varepsilon}$.

The debonding of the film is governed by Griffith's law [12], in terms of the dynamic energy release rate $G^{\varepsilon}$. In a general two-dimensional context, where $w^{\varepsilon}: \mathbb{R}^{2} \rightarrow$ $\mathbb{R}^{2}$ denotes the displacement, $G^{\varepsilon}$ is defined as the limit of a path integral where the path $C_{r}$ tends to the tip of the crack as $r \rightarrow 0$ (see [11]):

$$
\begin{aligned}
G^{\varepsilon}:=\lim _{r \rightarrow 0} \int_{C_{r}} & ( \\
& \left(\frac{\rho}{2}\left|\frac{\partial w^{\varepsilon}}{\partial t}\right|^{2}+\frac{N}{2}\left|\nabla w^{\varepsilon}\right|^{2}\right) n_{1} \\
& \left.-\frac{\partial w^{\varepsilon}}{\partial x_{1}} \cdot\left(\nabla w^{\varepsilon} n\right)\right) \mathrm{d} s
\end{aligned}
$$

where $n$ denotes the outer unit normal to $C_{r}$ and $s$ stands for the arc length parameter. Notice that the term containing the time derivative in (23) refers to the kinetic energy, while the others correspond to the static energy release rate.

In the one-dimensional context used in this paper, $C_{r}$ reduces to two points approaching the debonding tip from the two opposite sides, so, taking into account the orientation of the debonding front, (23) simplifies as follows:

$G^{\varepsilon}:=\frac{N}{2}\left(\llbracket\left(\omega^{\varepsilon}\right)^{2} \rrbracket-\varepsilon^{2} \llbracket\left(v^{\varepsilon}\right)^{2} \rrbracket\right)=\frac{N}{2}\left(1-\left(\varepsilon \dot{\ell}^{\varepsilon}\right)^{2}\right)\left(\omega_{+}^{\varepsilon}\right)^{2}$

(we refer to $[7$, Appendix A] for more details). In the last equality we dropped the dependence on $(T, \ell)$ and we used (4) and (22). Griffith's dynamic law finally reads

$\dot{\ell}^{\varepsilon} \geqslant 0, \quad \mathrm{G}^{\varepsilon} \leqslant \mathrm{G}_{\mathrm{c}}\left(\ell^{\varepsilon}\right), \quad\left(\mathrm{G}^{\varepsilon}-\mathrm{G}_{\mathrm{c}}\left(\ell^{\varepsilon}\right)\right) \dot{\ell}^{\varepsilon}=0$.

Notice that, according to (24) and (25), the speed of debonding is necessarily smaller than the wave speed:

$0 \leqslant \varepsilon \dot{\ell}^{\varepsilon}<1$

Following [7], we assume that the speed of debonding and the fields of infinitesimal rotation and transverse velocity are constant in each of the sectors delimited by the shock waves and by the front of debonding. This assumption seems reasonable, because

- both boundary and limit conditions are constant,

- the problem is linear (except of course for the propagation law), and

- the shock waves propagate with a velocity equal to $\pm 1 / \varepsilon$. 
Equations (17)-(25) permit then to perform the complete dynamic analysis of the debonding evolution.

Both in cases (a) and (b) (see (10) and (11)), the debonding front propagates at a constant speed, until it reaches the discontinuity of the toughness. The change of toughness generates a backward shock wave and modifies the speed of debonding. Later, the backward shock wave is reflected on the axis $x=0$ and transformed into a forward shock wave, which intersects the front of debonding, since the wave speed is greater than the one of debonding. This leads to a further change in the speed of debonding, while the forward shock wave is reflected into a backward one, and so on.

In [7] it is shown that, in both cases, the dynamic solutions converge to the quasistatic one (described in the previous section) as the speed of loading tends to zero. This is easier for case (a), since Griffith's classical dynamic law converges towards the usual quasistatic one in cases of stable propagation. On the contrary, more attention must be paid to case (b), when Griffith's classical quasistatic law fails. In case (b), it can be seen that the jump in the quasistatic solution is the limit of a dynamic fast propagation, corresponding to one backward/forward transition of the shock wave; during this process, the kinetic energy increases and suddenly vanishes. Hence one obtains an unexpected result: the phase of unstable propagation can be approximated by means of quasistatic quantities, because the kinetic energy plays only a transitory role. The correct criterion to predict the jump turns out to be the conservation of the total quasistatic energy.

\section{The case of a defect}

In the previous section, we presented the essential items for the quasistatic and the dynamical analysis of a peeling test. If the toughness has only one discontinuity, the dynamical solutions converge to the quasistatic one as the loading speed vanishes; hence, the kinetic energy plays a transitory role and does not need to be computed, since the value of the jump in crack length is given by the conservation of the total quasistatic energy.

We consider now the same peeling test in the case of a defect: the toughness is assumed to have two discontinuities. Here, the kinetic energy turns out to be relevant, so that the dynamic solutions do not converge to the quasistatic one (Proposition 6). This leads to the definition of an equivalent quasistatic toughness, whose expression is provided in (78).

\subsection{Peeling test with two discontinuities}

As in Section 1, we consider a peeling test for a film, bonded to a substrate with a specific toughness repartition, subject to a tension $N$ and a given opening displacement field $W$; due to the loading, the front of debonding propagates.

We assume now that the toughness has the following distribution:

$G_{c}(x)= \begin{cases}N \gamma_{2} & \text { if } 0<x \leqslant \ell_{1}, \\ N \gamma_{1} & \text { if } \ell_{1}<x \leqslant \ell_{2}, \\ N \gamma_{2} & \text { if } x>\ell_{2},\end{cases}$

where $0<\ell_{1}<\ell_{2}$ and $0<\gamma_{1}<\gamma_{2}$ again. This corresponds to a defect, i.e., the inclusion of particles whose toughness is smaller than the one of the matrix.

Remark 1 We do not treat the case of a defect with toughness greater than the matrix (that is, $G_{c}(x)=$ $N \gamma_{2}$ for $\ell_{1}<x \leqslant \ell_{2}, G_{c}(x)=N \gamma_{1}$ otherwise). In fact, this situation does not present a particular behaviour: the first discontinuity (increase of toughness) causes a temporary arrest of the debonding, until the loading overtakes a certain threshold. When the propagation continues and the second discontinuity is reached, the effect of the first one is somehow "forgotten", so we have the usual situation of decreasing toughness. The global solution is thus a simple combination of the two cases analysed in [7] and summarized in Section 1; the dynamic solutions converge to the quasistatic one as the loading speed tends to zero.

In the following sections we compare the quasistatic and the dynamic solutions as the loading speed tends to zero, for a defect of the type (27).

\subsection{Quasistatic solution}

Following the lines of [7] (see Section 1), the quasistatic evolution is supposed to be governed by Griffith's law when possible, and by a criterion of conservation of the total quasistatic energy otherwise.

During the first phase, i.e., before the first discontinuity $\left(\ell \leqslant \ell_{1}\right)$, Griffith's law admits a unique solution: by $(7),(9)$, and $(27)$ one has

$\ell(T)=\frac{T}{\sqrt{2 \gamma_{2}}}$ for $0 \leqslant T \leqslant \ell_{1} \sqrt{2 \gamma_{2}}$.

The decrease in toughness at the first discontinuity $(\ell=$ $\ell_{1}, T=T_{1}:=\ell_{1} \sqrt{2 \gamma_{2}}$ ) leads to an unstable propagation of the debonding (see Section 1). For this level of 
loading, the quasistatic energy (8) reads

$$
\begin{aligned}
& \mathcal{E}^{q}\left(T_{1}, \ell\right)= \\
& \begin{cases}N L\left(\frac{\ell_{1}^{2} \gamma_{2}}{\ell}+\ell \gamma_{2}\right) & \text { if } 0<\ell \leqslant \ell_{1}, \\
N L\left(\frac{\ell_{1}^{2} \gamma_{2}}{\ell}+\ell_{1} \gamma_{2}+\left(\ell-\ell_{1}\right) \gamma_{1}\right) & \text { if } \ell_{1}<\ell \leqslant \ell_{2}, \\
N L\left(\frac{\ell_{1}^{2} \gamma_{2}}{\ell}+\ell_{1} \gamma_{2}\right. & \text { if } \ell>\ell_{2} .\end{cases}
\end{aligned}
$$

The jump in crack length is determined by the criterion of energy conservation and the assumption of crack irreversibility. Calling $\ell_{b}>1$ the position of the debonding front at the end of the unstable phase, these conditions give

$\mathcal{E}^{q}\left(T_{1}, \ell_{b}\right)=\mathcal{E}^{q}\left(T_{1}, \ell_{1}\right)=2 N L \ell_{1} \gamma_{2}$.

In presence of a second discontinuity at $\ell_{2}>\ell_{1}$, two cases are possible. They depend on the value of $\ell_{c}$, which is defined as the length of the debonding front after the unstable phase if the toughness presents only one discontinuity (as seen above, $\ell_{c}=\ell_{1} \frac{\gamma_{2}}{\gamma_{1}}>\ell_{1}$ ). The two cases are given by

Case I (long defect): $\ell_{c} \leqslant \ell_{2}$,

Case II (small defect): $\ell_{c}>\ell_{2}$.

In case $I$, the unstable phase stops at $\ell_{b}=\ell_{c} \leqslant \ell_{2}$, in other words inside the defect. By (7) and (27) the energy release rate is

$G\left(T_{1}, \ell_{c}\right)=N \frac{\gamma_{1}^{2}}{\gamma_{2}}<N \gamma_{1}=G_{c}\left(\ell_{c}\right)$

Therefore, as predicted by Griffith's law (9), the debonding front stops until the loading $T$ reaches the value $\widetilde{T}_{c}$ such that $G\left(\widetilde{T}_{c}, \ell_{c}\right)=G_{c}\left(\ell_{c}\right)=N \gamma_{1}$. By (7) we have

$\widetilde{T}_{c}=\ell_{1} \frac{2 \gamma_{2}}{\sqrt{2 \gamma_{1}}}$

Between $\ell_{c}$ and $\ell_{2}$ (if the interval is not empty) the debonding is given by Griffith's law:

$\ell(T)=\frac{T}{\sqrt{2 \gamma_{1}}}$ for $\widetilde{T}_{c}<T \leqslant T_{2}$,

PSfrag replacements

where $T_{2}:=\ell_{2} \sqrt{2 \gamma_{1}}$. In order to study the second discontinuity, it is sufficient to recall the simple case of increasing toughness (Section 1): the debonding length remains constant $\left(\ell(T)=\ell_{2}\right)$ as long as the opening $T$ is less than $\widetilde{T}_{2}$, which is defined as the loading such that the energy release rate is equal to $N \gamma_{2}$. By (7) we obtain

$\widetilde{T}_{2}=\ell_{2} \sqrt{2 \gamma_{2}}$.
Then, when $T>\widetilde{T}_{2}$, the debonding restarts again following Griffith's law with $G_{c}=N \gamma_{2}$.

This concludes the analysis of case I, under the assumption that the debonding evolution is determined by Griffith's law except during the unstable phase, where the jump is governed by the principle of quasistatic energy conservation. In case I, the map $T \mapsto \ell(T)$ is given by

$$
\ell(T)= \begin{cases}\frac{T}{\sqrt{2 \gamma_{2}}} & \text { if } 0 \leqslant T \leqslant \ell_{1} \sqrt{2 \gamma_{2}}, \\ \ell_{c}=\frac{\gamma_{2}}{\gamma_{1}} \ell_{1} & \text { if } \ell_{1} \sqrt{2 \gamma_{2}}<T \leqslant \ell_{1} \frac{2 \gamma_{2}}{\sqrt{2 \gamma_{1}}}, \\ \frac{T}{\sqrt{2 \gamma_{1}}} & \text { if } \ell_{1} \frac{2 \gamma_{2}}{\sqrt{2 \gamma_{1}}}<T \leqslant \ell_{2} \sqrt{2 \gamma_{1}}, \\ \ell_{2} & \text { if } \ell_{2} \sqrt{2 \gamma_{1}}<T \leqslant \ell_{2} \sqrt{2 \gamma_{2}}, \\ \frac{T}{\sqrt{2 \gamma_{2}}} & \text { if } T>\ell_{2} \sqrt{2 \gamma_{2}}\end{cases}
$$

(see Figure 3).

Case II corresponds to the situation where the size of the defect is small $\left(\ell_{c}>\ell_{2}\right)$. Hence, the criterion of energy conservation does not admit any solution inside the defect: it is not possible to find any $\ell_{b} \in\left(\ell_{1}, \ell_{2}\right]$ such that $\mathcal{E}^{q}\left(T_{1}, \ell_{b}\right)=\mathcal{E}^{q}\left(T_{1}, \ell_{1}\right)$. Then, as one can easily see by solving the last equation, the unstable crack propagation phase stops outside the defect, at length

$$
\begin{aligned}
\ell_{b}=\frac{1}{2 \gamma_{2}}( & \gamma_{2}\left(\ell_{2}+\ell_{1}\right)-\gamma_{1}\left(\ell_{2}-\ell_{1}\right) \\
& \left.+\sqrt{\left(\gamma_{2}\left(\ell_{2}+\ell_{1}\right)-\gamma_{1}\left(\ell_{2}-\ell_{1}\right)\right)^{2}-4 \ell_{1}^{2} \gamma_{2}^{2}}\right)
\end{aligned}
$$

with $\ell_{b}>\ell_{2}$. Since $\mathcal{E}^{q}\left(T_{1}, \ell_{b}\right)=\mathcal{E}^{q}\left(T_{1}, \ell_{1}\right)<\mathcal{E}^{q}\left(T_{1}, \ell_{c}\right)$, we have $\ell_{b}<\ell_{c}$. As for the energy release rate, it turns out that

$G\left(T_{1}, \ell_{b}\right)<N \gamma_{2}=G\left(T_{1}, \ell_{1}\right)=G_{c}\left(\ell_{b}\right)$.

Therefore, by Griffith's law (9) the debonding front stops until the loading $T$ reaches the value $\widetilde{T}_{b}$ such that 
$G\left(\widetilde{T}_{b}, \ell_{b}\right)=G_{c}\left(\ell_{b}\right)=N \gamma_{2}$. By $(7)$ we have

$\widetilde{T}_{b}=\ell_{b} \sqrt{2 \gamma_{2}}$

As $T>\widetilde{T}_{b}$, the debonding restarts again following Griffith's law with $G_{c}=N \gamma_{2}$. Hence, in case II the evolution of the debonding length $\ell$ is given by

$\ell(T)= \begin{cases}\frac{T}{\sqrt{2 \gamma_{2}}} & \text { if } 0 \leqslant T \leqslant \ell_{1} \sqrt{2 \gamma_{2}}, \\ \ell_{b} & \text { if } \ell_{1} \sqrt{2 \gamma_{2}}<T \leqslant \ell_{b} \sqrt{2 \gamma_{2}}, \\ \frac{T}{\sqrt{2 \gamma_{2}}} & \text { if } T>\ell_{b} \sqrt{2 \gamma_{2}}\end{cases}$

(see Figure 3).

The quasistatic analysis of a defect with toughness lower than the matrix exhibits two main features:

- the length of the jump of the debonding can take two different values depending on the size of the defect: if it is large, the crack stops inside the defect, otherwise the cracks passes through and stops outside the defect if it is small;

- no memory effect is put forward: after the unstable phase due to the defect, the propagation remains stable and follows Griffith's classical law as if the defect did not exist.

The next step consists in performing the dynamic analysis and comparing the results.

\subsection{Dynamic analysis}

In this section, we analyse the dynamics of the peeling test containing one defect, presented above. As in section 1.3, each change of toughness generates a backward shock wave, which is reflected on the axis $x=0$ and transformed into a forward shock wave intersecting the front of debonding and modifying again the crack speed. Since there are two points of discontinuity for the toughness, we deal now with two different groups of shock waves.

In the same spirit as in [7], the $(x, T)$-plane is divided into sectors $\mathcal{Q}_{i}^{\varepsilon}, i \geqslant 0$, delimited either by the shock waves, or by the coordinate axes. As defined above, the sector $\mathcal{Q}_{0}^{\varepsilon}$ is delimited by the front of debonding and the axis $T=0$, and corresponds to the region with $\omega^{\varepsilon}=v^{\varepsilon}=0$. The sector $\mathcal{Q}_{1}^{\varepsilon}$ is delimited by the front of debonding, the backward shock wave generated at $\ell_{1}$, and the axis $x=0$; then the index $i$ is incremented at each reflection of the shock waves, as shown in Figure 4 . The infinitesimal rotation $\omega^{\varepsilon}$ and the transverse velocity $v^{\varepsilon}$ are assumed to be constant on each sector; the symbols $\omega_{i}^{\varepsilon}$ and $v_{i}^{\varepsilon}$ denote the values of $\omega^{\varepsilon}$ and $v^{\varepsilon}$ on the sector $\mathcal{Q}_{i}^{\varepsilon}$, respectively.

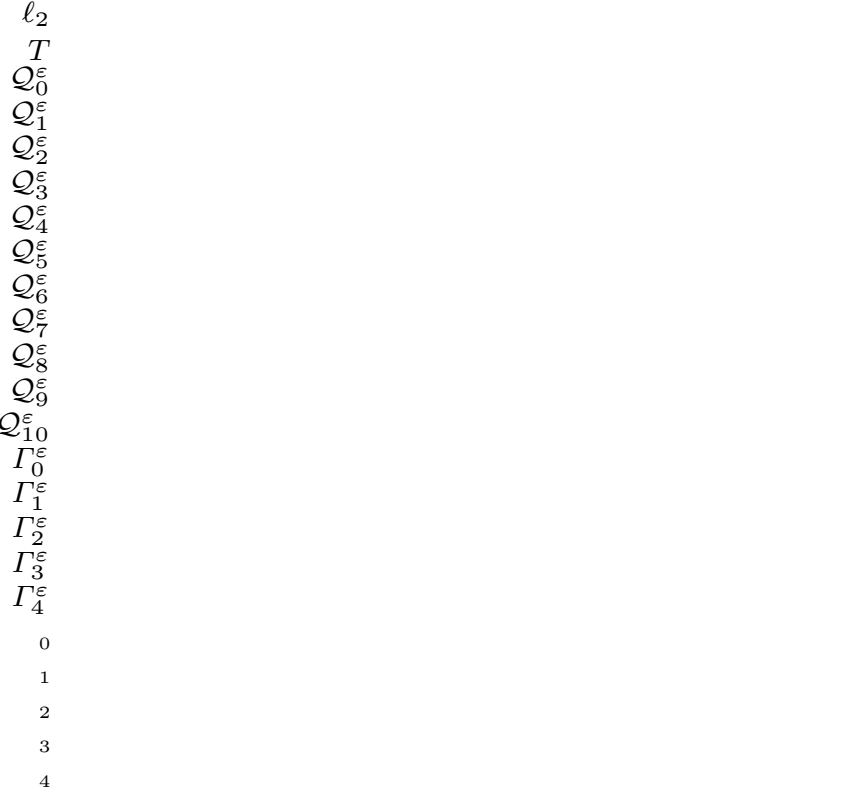

Fig. 4 Sectors in the $(x, T)$-plane: the thick line represents the debonding, the thin ones are the shock waves. The numbers $0, \ldots, 4$ denote, respectively, the points $\left(\ell_{0}^{\varepsilon}, T_{0}^{\varepsilon}\right), \ldots,\left(\ell_{4}^{\varepsilon}, T_{4}^{\varepsilon}\right)$.

The long defect (case I) is not of particular interest. The kinetic energy generated during the unstable propagation is totally relaxed before the debonding reaches the second discontinuity: indeed, $\ell_{c}$ corresponds to the length where the kinetic energy vanishes (see [7]). This case can thus be seen as a combination of cases (a) and (b) of Section 1, disconnected in the sense of the role of the kinetic energy; therefore, the dynamic solutions converge to the quasistatic one (under the principle of energy conservation). Both the dynamic and the quasistatic solutions for case I are presented in Figure 5.

We consider here only the small defect (case II). Since $\ell_{c}>\ell_{2}$, it is easy to see that the debonding reaches the second discontinuity before being intersected by the first forward shock wave. Hence, the kinetic energy is not totally relaxed when the debonding reaches the second discontinuity. We will study the consequence of this residual kinetic energy on the propagation of the debonding.

The front of debonding $\Gamma^{\varepsilon}$ is decomposed into linear segments $\Gamma_{i}^{\varepsilon}, i \geqslant 0$, numbered in such a way that $\Gamma_{i}^{\varepsilon}$ stands between $\ell_{i}^{\varepsilon}$ and $\ell_{i+1}^{\varepsilon}$, where $\ell_{0}^{\varepsilon}:=0, \ell_{1}^{\varepsilon}:=\ell_{1}$, $\ell_{2}^{\varepsilon}:=\ell_{2}$, and $\ell_{i}^{\varepsilon}$ corresponds to the $i$-th reflection of waves (see Figure 4 ). We shall determine the speed $\dot{\ell}_{i}^{\varepsilon}$ of the front of debonding, that is the slope of $\Gamma_{i}^{\varepsilon}$, taking into account the kinetic energy. For this purpose, we shall compute the fields $\omega_{i}^{\varepsilon}$ and $v_{i}^{\varepsilon}$ in the different sectors and the dynamic energy release rate $G_{i}^{\varepsilon}$ on $\Gamma_{i}^{\varepsilon}$. 


$$
\ell_{1}=
$$

Fig. 5 Convergence of the dynamic solutions to the quasistatic one in the case of a long defect (case I). Here, $\gamma_{1}=0.5, \gamma_{2}=2$, $\ell_{1}=1, \ell_{2}=5$.

First, we will use the tools of Section 1.3 (more precisely, equations (21)-(25)) in order to describe the evolution $\ell^{\varepsilon}$. Then, we will study the evolution of the kinetic energy, which shows quite a complex behaviour.

\subsubsection{Initial stage}

While $\ell \leqslant \ell_{2}$, only one discontinuity of the toughness affects the solution, so it is sufficient to follow [7] . Before the first discontinuity, the boundary condition (20), the Hadamard compatibility condition (21) and Griffith's dynamic law (25) give

$\dot{\ell}_{0}^{\varepsilon}=\frac{1}{\sqrt{2 \gamma_{2}+\varepsilon^{2}}}$.

The first discontinuity $\left(\ell=\ell_{1}\right)$ is thus reached at the load

$T_{1}^{\varepsilon}:=\ell_{1} \sqrt{2 \gamma_{2}+\varepsilon^{2}}$.

Afterwards, the debonding proceeds with speed

$\dot{\ell}_{1}^{\varepsilon}=\frac{1}{\varepsilon} \frac{\left(\sqrt{2 \gamma_{2}+\varepsilon^{2}}+\varepsilon\right)^{2}-2 \gamma_{1}}{\left(\sqrt{2 \gamma_{2}+\varepsilon^{2}}+\varepsilon\right)^{2}+2 \gamma_{1}}$

and the second discontinuity $\left(\ell=\ell_{2}\right)$ is reached for $T=T_{2}^{\varepsilon}$, with

$T_{2}^{\varepsilon}:=T_{1}^{\varepsilon}+\varepsilon \frac{\left(\sqrt{2 \gamma_{2}+\varepsilon^{2}}+\varepsilon\right)^{2}+2 \gamma_{1}}{\left(\sqrt{2 \gamma_{2}+\varepsilon^{2}}+\varepsilon\right)^{2}-2 \gamma_{1}}\left(\ell_{2}-\ell_{1}\right)$.
As $\varepsilon \rightarrow 0, T_{1}^{\varepsilon}$ and $T_{2}^{\varepsilon}$ tend to $T_{1}=\ell_{1} \sqrt{2 \gamma_{2}}$, while $\dot{\ell}_{1}^{\varepsilon}$ diverges (indeed, the limit of $\ell^{\varepsilon}$ has a jump).

Using [7, Proposition 3.1] we find the fields of infinitesimal rotation and transverse velocity:

$\left\{\begin{array}{l}v_{1}^{\varepsilon}=1, \\ \omega_{1}^{\varepsilon}=-\sqrt{2 \gamma_{2}+\varepsilon^{2}},\end{array}\right.$

$\left\{\begin{array}{l}v_{2}^{\varepsilon}=-\dot{\ell}_{1}^{\varepsilon} \omega_{2}^{\varepsilon}, \\ \omega_{2}^{\varepsilon}=-\frac{\gamma_{1}+\gamma_{2}}{\sqrt{2 \gamma_{2}+\varepsilon^{2}}+\varepsilon}-\varepsilon\end{array}\right.$

$\left\{\begin{array}{l}v_{3}^{\varepsilon}=1, \\ \omega_{3}^{\varepsilon}=-\frac{2 \gamma_{1}}{\sqrt{2 \gamma_{2}+\varepsilon^{2}}+\varepsilon}-\varepsilon .\end{array}\right.$

We will use these fields for the computation of the velocity of propagation after the second discontinuity.

\subsubsection{Intermediate stage}

When the second discontinuity is reached, the second shock wave is created, dividing the plane in sectors $\mathcal{Q}_{i}^{\varepsilon}$ as described above. The fields of infinitesimal rotation and transverse velocity in each sector can then be computed inductively using (21). The propagation speeds $\dot{\ell}_{m}^{\varepsilon}, \dot{\ell}_{m+1}^{\varepsilon}, \ldots$ are determined by the mechanical fields in the corresponding sectors $\mathcal{Q}_{3 m-2}^{\varepsilon}, \mathcal{Q}_{3 m+1}^{\varepsilon} \ldots$, which are coupled with the sectors $\mathcal{Q}_{3 m}^{\varepsilon}, \mathcal{Q}_{3 m+3}^{\varepsilon} \ldots$ If the sectors $\mathcal{Q}_{i}^{\varepsilon}$ are known for every $i \leqslant 3 m$, the values in the following sectors are given by the next proposition, which is the counterpart of [7, Proposition 3.1]. 
Proposition 2 For every $m \geqslant 2$ we have

$\left\{\begin{array}{l}v_{3 m+1}^{\varepsilon}=-\dot{\ell}_{m+1}^{\varepsilon} \omega_{3 m+1}^{\varepsilon}, \\ \omega_{3 m+1}^{\varepsilon}=\frac{\omega_{3 m-3}^{\varepsilon}-\varepsilon}{1+\varepsilon \dot{\ell}_{m+1}^{\varepsilon}}, \\ \dot{\ell}_{m+1}^{\varepsilon}=\frac{1}{\varepsilon} \frac{\left(\left(\omega_{3 m-3}^{\varepsilon}-\varepsilon\right)^{2}-2 \gamma_{2}\right)^{+}}{\left(\omega_{3 m-3}^{\varepsilon}-\varepsilon\right)^{2}+2 \gamma_{2}},\end{array}\right.$

$\left\{\begin{array}{l}v_{3 m+3}^{\varepsilon}=1 \\ \omega_{3 m+3}^{\varepsilon}=2 \omega_{3 m+1}^{\varepsilon}-\omega_{3 m-3}^{\varepsilon},\end{array}\right.$

where $(\cdot)^{+}$stands for the positive part. For $m=1$ the previous relations hold substituting $\omega_{3 m-3}$ with $\omega_{1}$.

Proof The proof follows the lines of [7, Proposition 3.1]. By (22), (24),(25), and by $(21)_{2}$ between $\mathcal{Q}_{3 m+1}^{\varepsilon}$ and $\mathcal{Q}_{3 m-1}^{\varepsilon}$ and between $\mathcal{Q}_{3 m-1}^{\varepsilon}$ and $\mathcal{Q}_{3 m-3}^{\varepsilon}$, we get for every $m \geqslant 2$

$v_{3 m+1}^{\varepsilon}=-\dot{\ell}_{m+1}^{\varepsilon} \omega_{3 m+1}^{\varepsilon}$,

$\omega_{3 m+1}^{\varepsilon}-\omega_{3 m-3}^{\varepsilon}=\varepsilon\left(v_{3 m+1}^{\varepsilon}-v_{3 m-3}^{\varepsilon}\right)$,

$G_{m+1}^{\varepsilon}=\frac{N}{2}\left(1-\left(\varepsilon \dot{\ell}_{m+1}^{\varepsilon}\right)^{2}\right)\left(\omega_{3 m+1}^{\varepsilon}\right)^{2} \leqslant N \gamma_{2}$,

where (53) is actually an equality if $\dot{\ell}_{m+1}^{\varepsilon} \neq 0$. For $m=1$, these expressions still hold substituting $\mathcal{Q}_{3 m-3}^{\varepsilon}$ with $\mathcal{Q}_{1}^{\varepsilon}$.

By (51) and (52) we get $\omega_{3 m+1}^{\varepsilon}=\frac{\omega_{3 m-3}^{\varepsilon}-\varepsilon}{1+\varepsilon \dot{\ell}_{m+1}^{\varepsilon}}$. If $\dot{\ell}_{m+1}^{\varepsilon} \neq$ 0 , substituting $\omega_{3 m+1}^{\varepsilon}$ in (53) we find

$$
\dot{\ell}_{m+1}^{\varepsilon}=\frac{1}{\varepsilon} \frac{\left(\omega_{3 m-3}^{\varepsilon}-\varepsilon\right)^{2}-2 \gamma_{2}}{\left(\omega_{3 m-3}^{\varepsilon}-\varepsilon\right)^{2}+2 \gamma_{2}}
$$

In the opposite case, by (53) we obtain $\left(\omega_{3 m-3}^{\varepsilon}-\varepsilon\right)^{2} \leqslant$ $2 \gamma_{2}$.

Recalling (20), we have $v_{3 m+3}^{\varepsilon}=1$. Using again (21) we get

$$
\begin{gathered}
\omega_{3 m-3}^{\varepsilon}-\omega_{3 m+1}^{\varepsilon}=\varepsilon\left(1-v_{3 m+1}^{\varepsilon}\right), \\
\omega_{3 m+3}^{\varepsilon}-\omega_{3 m+1}^{\varepsilon}=-\varepsilon\left(1-v_{3 m+1}^{\varepsilon}\right) .
\end{gathered}
$$

Summing up in the last two equations, we conclude.

The previous formulas show that the sectors $\mathcal{Q}_{3 m+1}$ are divided in two uncoupled families, according as $m$ is even or odd. If $m$ is even, we set $n:=m / 2$ and find the family of sectors of the type $\mathcal{Q}_{6 n-2}^{\varepsilon}$, coupled with $\mathcal{Q}_{6 n}^{\varepsilon}$; if $m$ is odd, for $n:=(m-1) / 2$ we obtain the family $\mathcal{Q}_{6 n+1}^{\varepsilon}$, coupled with $\mathcal{Q}_{6 n+3}^{\varepsilon}$. Starting from (46)-(48), we compute the values of the mechanical fields, until a certain threshold is overtaken.
Proposition 3 For every $\varepsilon>0$ there exists an integer $N^{\varepsilon}$ such that for every $n$ such that $1 \leqslant n<N^{\varepsilon}$ we have

$\left\{\begin{array}{l}v_{6 n-2}^{\varepsilon}=1, \\ \omega_{6 n-2}^{\varepsilon}=-\sqrt{2 \gamma_{2}+\varepsilon^{2}}, \\ \dot{\ell}_{2 n}^{\varepsilon}=\dot{\ell}_{0}^{\varepsilon},\end{array}\right.$
$\left\{\begin{array}{l}v_{6 n}^{\varepsilon}=1, \\ \omega_{6 n}^{\varepsilon}=-\sqrt{2 \gamma_{2}+\varepsilon^{2}},\end{array}\right.$
$\left\{\begin{array}{l}v_{6 n+1}^{\varepsilon}=0, \\ \omega_{6 n+1}^{\varepsilon}=-\frac{2 \gamma_{1}}{\sqrt{2 \gamma_{2}+\varepsilon^{2}}+\varepsilon}-2 n \varepsilon, \\ \dot{\ell}_{2 n+1}^{\varepsilon}=0,\end{array}\right.$

$\left\{\begin{array}{l}v_{6 n+3}^{\varepsilon}=1, \\ \omega_{6 n+3}^{\varepsilon}=-\frac{2 \gamma_{1}}{\sqrt{2 \gamma_{2}+\varepsilon^{2}}+\varepsilon}-(2 n+1) \varepsilon .\end{array}\right.$

Proof Let us start with $n=1$ and then argue by induction. Since $\dot{\ell}_{2}^{\varepsilon} \neq 0$, by (24) and (25) we have $N \gamma_{2}=$ $G_{1}^{\varepsilon}<\frac{1}{2} \omega_{1}^{2}$. If $\dot{\ell}_{2}^{\varepsilon}=0$, by (46)-(48) and (49)-(50) we obtain $\frac{1}{2} \omega_{1}^{2}<\frac{1}{2} \omega_{4}^{2}$; on the other hand $\frac{1}{2} \omega_{4}^{2}=G_{2}^{\varepsilon} \leqslant N \gamma_{2}$, that is a contradiction. Hence, $\dot{\ell}_{2}^{\varepsilon} \neq 0$ and we find a system of three equalities for $\mathcal{Q}_{4}^{\varepsilon}$, which show

$$
\begin{gathered}
v_{4}^{\varepsilon}=v_{1}^{\varepsilon}=1, \quad \omega_{4}^{\varepsilon}=\omega_{1}^{\varepsilon}=-\sqrt{2 \gamma_{2}+\varepsilon^{2}}, \\
\dot{\ell}_{2}^{\varepsilon}=\dot{\ell}_{0}^{\varepsilon}=\frac{1}{\sqrt{2 \gamma_{2}+\varepsilon^{2}}} .
\end{gathered}
$$

By (49)-(50) we get $v_{6}^{\varepsilon}=1$ and $\omega_{6}^{\varepsilon}=\omega_{1}^{\varepsilon}$.

Repeating the argument inductively, we find for every $n \geqslant 1$

$$
v_{6 n}^{\varepsilon}=v_{6 n-2}^{\varepsilon}=1, \quad \omega_{6 \mathrm{n}}^{\varepsilon}=\omega_{6 \mathrm{n}-2}^{\varepsilon}=\omega_{1}^{\varepsilon}, \quad \dot{\ell}_{2 \mathrm{n}}^{\varepsilon}=\dot{\ell}_{0}^{\varepsilon} .
$$

This completes the proof for $\mathcal{Q}_{6 n-2}^{\varepsilon}$ and $\mathcal{Q}_{6 n}^{\varepsilon}$.

Let us pass to $\mathcal{Q}_{6 n+1}^{\varepsilon}$ and $\mathcal{Q}_{6 n+3}^{\varepsilon}$. For $n=1$, assume by contradiction that $\dot{\ell}_{3}^{\varepsilon} \neq 0$; then using (24), (25), (46)-(48), and (49)-(50) we get $G_{3}^{\varepsilon}<\frac{N}{2}\left(\omega_{7}^{\varepsilon}\right)^{2}<$ $\frac{N}{2}\left(\omega_{3}^{\varepsilon}-\varepsilon\right)^{2} \leqslant N \gamma_{2}$, which implies that $\dot{\ell}_{3}^{\varepsilon}=0$. Hence, $\dot{\ell}_{3}^{\varepsilon}=0$ and $v_{3}^{\varepsilon}=0$.

Repeating the scheme, we compute by induction $\omega_{6 n+1}^{\varepsilon}, v_{6 n+1}^{\varepsilon}$, and $\omega_{6 n+3}^{\varepsilon}$ for all indices $n$ such that $\left(\omega_{6 n-3}^{\varepsilon}-\varepsilon\right)^{2} \leqslant 2 \gamma_{2}$. In particular, $\dot{\ell}_{2 n+1}^{\varepsilon}=0$ for those indices.

Remark 4 The last proposition shows the repetition of identical sectors of the type $\mathcal{Q}_{6 n-2}^{\varepsilon}$ and $\mathcal{Q}_{6 n}^{\varepsilon}$ (until $n<$ $N^{\varepsilon}$ ). Indeed, the shock wave generated when the debonding reaches the second discontinuity, restores the fields of rotation and velocity when intersecting the debonding. This gives some kind of "impulsion" to the crack, which is forced to propagate at Griffith's classical speed in a homogeneous media of thoughness $\gamma_{2}$, i.e., $\dot{\ell}_{0}^{\varepsilon}$. 
Instead, the shock wave generated at the first discontinuity stops the crack propagation when intersecting the debonding and increments the values of the rotation and the deformation fields in the sectors $\mathcal{Q}_{6 n+1}^{\varepsilon}$ and $\mathcal{Q}_{6 n+3}^{\varepsilon}$. This corresponds to the behaviour of the shock wave in the case of one discontinuity, which is here alternating with the "restoring" shock wave.

Remark 5 As shown in the proof, the best value for $N^{\varepsilon}$ is the least integer such that $\left(\omega_{6 N^{\varepsilon}-3}^{\varepsilon}-\varepsilon\right)^{2}>2 \gamma_{2}$. Using the expression for $\omega_{6 n-3}^{\varepsilon}$ we get

$N^{\varepsilon}=\left[\frac{1}{\varepsilon}\left(\sqrt{\frac{\gamma_{2}}{2}}-\frac{\gamma_{1}}{\sqrt{2 \gamma_{2}+\varepsilon^{2}}+\varepsilon}\right)\right]+1$,

where $[\cdot]$ denotes the integer part. Moreover, we have

$\lim _{\varepsilon \rightarrow 0} \varepsilon N^{\varepsilon}=\frac{\gamma_{2}-\gamma_{1}}{\sqrt{2 \gamma_{2}}}$.

This intermediate phase is consequently composed of alterning propagation and arrest phases. According to (59), the slower the loading, the larger the number of phases.

We now define, for $i=2, \ldots, 2 N^{\varepsilon}$, the points $\left(\ell_{i}^{\varepsilon}, T_{i}^{\varepsilon}\right)$ of intersection of the two shock waves with the debonding in the $(x, T)$-plane (see Figure 4$)$. We start from $\ell_{2}^{\varepsilon}:=\ell_{2}$ and $T_{2}^{\varepsilon}$ as in (45); it turns out that (for $n=$ $\left.1, \ldots, N^{\varepsilon}\right)$

$\left\{\begin{array}{l}\ell_{2 n+1}^{\varepsilon}=\ell_{2 n}^{\varepsilon}+\dot{\ell}_{0}^{\varepsilon}\left(T_{2 n+1}^{\varepsilon}-T_{2 n}^{\varepsilon}\right), \\ \ell_{2 n}^{\varepsilon}=\ell_{2 n-1}^{\varepsilon}, \\ T_{2 n+1}^{\varepsilon}=T_{2 n-1}^{\varepsilon}+\varepsilon\left(\ell_{2 n-1}^{\varepsilon}+\ell_{2 n+1}^{\varepsilon}\right), \\ T_{2 n}^{\varepsilon}=T_{2 n-1}^{\varepsilon}+\Delta^{\varepsilon},\end{array}\right.$

where

$\Delta^{\varepsilon}:=\left(\ell_{2}-\ell_{1}\right) \varepsilon \frac{2\left(\sqrt{2 \gamma_{2}+\varepsilon^{2}}+\varepsilon\right)^{2}}{\left(\sqrt{2 \gamma_{2}+\varepsilon^{2}}+\varepsilon\right)^{2}-2 \gamma_{1}}$

denotes the variation of loading. Notice that $\Delta^{\varepsilon}$ is independent of $n$, it vanishes as $\varepsilon \rightarrow 0$, and

$\lim _{\varepsilon \rightarrow 0} \frac{\Delta^{\varepsilon}}{\varepsilon}=\left(\ell_{2}-\ell_{1}\right) \frac{2 \gamma_{2}}{\gamma_{2}-\gamma_{1}}$.

In order to determine the curve followed by the debonding, it is sufficient to know $\left(\ell_{2 n}^{\varepsilon}, T_{2 n}^{\varepsilon}\right)$ for $n=1, \ldots, N^{\varepsilon}$. Using the previous relations one sees that

$$
\begin{aligned}
& \ell_{2 n+2}^{\varepsilon}=\left(C_{1}^{\varepsilon}\right)^{n} \ell_{2}-C_{2}^{\varepsilon} \sum_{j=0}^{n-1}\left(C_{1}^{\varepsilon}\right)^{j}, \\
& T_{2 n+2}^{\varepsilon}=T_{2}^{\varepsilon}+n \Delta^{\varepsilon}+\frac{\ell_{2 n+2}^{\varepsilon}-\ell_{2}}{\dot{\ell}_{0}^{\varepsilon}},
\end{aligned}
$$

where

$$
\begin{gathered}
C_{1}^{\varepsilon}:=\frac{1+\varepsilon \dot{\ell}_{0}^{\varepsilon}}{1-\varepsilon \dot{\ell}_{0}^{\varepsilon}}=\frac{\sqrt{2 \gamma_{2}+\varepsilon^{2}}+\varepsilon}{\sqrt{2 \gamma_{2}+\varepsilon^{2}}-\varepsilon}, \\
C_{2}^{\varepsilon}:=\Delta^{\varepsilon} \frac{\dot{\ell}_{0}^{\varepsilon}}{1-\varepsilon \dot{\ell}_{0}^{\varepsilon}}=\frac{\Delta^{\varepsilon}}{\sqrt{2 \gamma_{2}+\varepsilon^{2}}-\varepsilon} .
\end{gathered}
$$

Notice that, as $\varepsilon \rightarrow 0$,

$$
\begin{aligned}
& C_{1}^{\varepsilon} \rightarrow 1, \quad \frac{1}{\varepsilon}\left(\mathrm{C}_{1}^{\varepsilon}-1\right) \rightarrow \sqrt{\frac{2}{\gamma_{2}}}, \\
& C_{2}^{\varepsilon} \rightarrow 0, \quad \frac{1}{\varepsilon} \mathrm{C}_{2}^{\varepsilon} \rightarrow\left(\ell_{2}-\ell_{1}\right) \frac{\sqrt{2 \gamma_{2}}}{\gamma_{2}-\gamma_{1}} .
\end{aligned}
$$

We now provide an analytic expression for the curve followed by the debonding during the intermediate stage. Rewriting (63) we have

$$
\ell_{2 n+2}^{\varepsilon}=\left(C_{1}^{\varepsilon}\right)^{n} \ell_{2}-C_{2}^{\varepsilon} \frac{\left(C_{1}^{\varepsilon}\right)^{n}-1}{C_{1}^{\varepsilon}-1},
$$

giving $\ell_{2 n+2}^{\varepsilon}$ in function of $n<N^{\varepsilon}$. We define an "inverse" of the previous relation, setting for every $\ell \in$ $\left[\ell_{2}, \ell_{2 N^{\varepsilon}}\right]$

$n^{\varepsilon}(\ell):=\left(\ln C_{1}^{\varepsilon}\right)^{-1} \ln \left(\frac{\ell\left(C_{1}^{\varepsilon}-1\right)-C_{2}^{\varepsilon}}{\ell_{2}^{\varepsilon}\left(C_{1}^{\varepsilon}-1\right)-C_{2}^{\varepsilon}}\right)$

and

$\bar{n}^{\varepsilon}(\ell):=\left[n^{\varepsilon}(\ell)\right]$.

Hence, $\bar{n}^{\varepsilon}(\ell)$ is the integer such that $\ell_{2 \bar{n}^{\varepsilon}(\ell)+2}^{\varepsilon} \leq \ell<$ $\ell_{2 \bar{n}^{\varepsilon}(\ell)+4}^{\varepsilon}$. For a fixed $\ell$, as $\varepsilon \rightarrow 0$ we have

$n^{\varepsilon}(\ell) \rightarrow+\infty$,

$\varepsilon n^{\varepsilon}(\ell) \rightarrow \sqrt{\frac{\gamma_{2}}{2}} \ln \left(\frac{\ell\left(\gamma_{2}-\gamma_{1}\right)-\left(\ell_{2}-\ell_{1}\right) \gamma_{2}}{\ell_{1} \gamma_{2}-\ell_{2} \gamma_{1}}\right)$,

and the same holds for $\bar{n}^{\varepsilon}(\ell)$, because $n^{\varepsilon}(\ell)-1 \leqslant$ $\bar{n}^{\varepsilon}(\ell) \leqslant n^{\varepsilon}(\ell)$ (we recall that $\ell_{1} \gamma_{2}-\ell_{2} \gamma_{1}>0$ since we are in case II; see (32)). Substituting $\bar{n}^{\varepsilon}(\ell)$ to $n$ in the expression for $T_{2 n+2}$ (63), we can parametrize the curve followed by the debonding as

$T^{\varepsilon}(\ell)=T_{2}^{\varepsilon}+\bar{n}^{\varepsilon}(\ell) \Delta^{\varepsilon}+\frac{\ell-\ell_{2}}{\dot{\ell}_{0}^{\varepsilon}}$

for $\ell \in\left[\ell_{2}, \ell_{2 N^{\varepsilon}}\right]$. As $\varepsilon \rightarrow 0$, this tends to the limit curve

$\ell \mapsto \sqrt{2 \gamma_{2}} \Phi(\ell)$,

with

$\Phi(\ell):=\ell-\left(\ell_{2}-\ell_{1}\right)\left(1-\frac{\gamma_{2}}{\gamma_{2}-\gamma_{1}} \phi(\ell)\right)$

and $\phi(\ell):=\ln \frac{\ell\left(\gamma_{2}-\gamma_{1}\right)-\left(\ell_{2}-\ell_{1}\right) \gamma_{2}}{\ell_{1} \gamma_{2}-\ell_{2} \gamma_{1}}$. Notice that $\Phi$ is an increasing concave function of $\ell$. 
$\ell_{1}=\quad \ell_{2} \quad \ell_{b} \quad \ell_{d}$

Fig. 6 Comparison between the dynamic solutions and the quasistatic one in the case of a short defect (case II). Here, $\gamma_{1}=0.5, \gamma_{2}=2, \ell_{1}=1, \ell_{2}=1.5$.

The quantity $\ell_{2 N^{\varepsilon}}^{\varepsilon}=\ell_{2 N^{\varepsilon}-1}^{\varepsilon}$ represents the debonding level reached at the end of the transition described in Proposition 3. Using (65) and (70) we see that

$\lim _{\varepsilon \rightarrow 0} \ell_{2 N^{\varepsilon}}^{\varepsilon}=\frac{\gamma_{2}\left(\ell_{2}-\ell_{1}\right)+\left(\ell_{1} \gamma_{2}-\ell_{2} \gamma_{1}\right) e^{1-\frac{\gamma_{1}}{\gamma_{2}}}}{\gamma_{2}-\gamma_{1}}=: \ell_{d}$.

The limit curve (72) joins at $\ell_{d}$ the classical quasistatic solution corresponding to the toughness $\gamma_{2}$ : indeed,

$\sqrt{2 \gamma_{2}} \Phi\left(\ell_{d}\right)=\sqrt{2 \gamma_{2}} \ell_{d}=: T_{d}$.

Since by assumption $\ell_{1} \gamma_{2}>\ell_{2} \gamma_{1}$, we have $\ell_{d}>$ $\ell_{2}$, so that the quasistatic solution is reached outside the defect. Fixed $\ell_{1}$ and letting $\ell_{2}$ vary between $\ell_{1}$ and $\ell_{1} \frac{\gamma_{2}}{\gamma_{1}}$, one sees that the distance $\ell_{d}-\ell_{2}$ decreases as $\ell_{2}$ increases; moreover,

$$
\begin{gathered}
\ell_{d}-\ell_{2}<\ell_{1} e^{1-\frac{\gamma_{1}}{\gamma_{2}}}, \\
\ell_{d}-\ell_{2} \rightarrow \ell_{1} e^{1-\frac{\gamma_{1}}{\gamma_{2}}} \quad \text { as } \ell_{2} \rightarrow \ell_{1}, \\
\ell_{d}-\ell_{2} \rightarrow 0 \text { as } \quad \ell_{2} \rightarrow \ell_{1} \frac{\gamma_{2}}{\gamma_{1}} .
\end{gathered}
$$

Finally, notice that $\ell_{c}>\ell_{d}$.

\subsubsection{Final stage}

For $n \geqslant N^{\varepsilon}$, the system switches to a different regime. The values of the mechanical fields are found by induction using again Proposition 2.

Actually, the equations for the sectors of the type $\mathcal{Q}_{6 n-2}^{\varepsilon}$ are not modified. Indeed, these sectors are independent of the ones of the type $\mathcal{Q}_{6 n+1}^{\varepsilon}$ and all the fields are constant up to $n=N^{\varepsilon}-1$. Hence, formulas (54)-(55) still hold, in particular $\dot{\ell}_{2 n}^{\varepsilon}=\dot{\ell}_{0}^{\varepsilon}$ for every $n$.

On the contrary, by the choice of $N^{\varepsilon}$ we find that $\dot{\ell}_{2 N^{\varepsilon}+1}^{\varepsilon} \neq 0$, which represents a change of regime in the sectors of the type $\mathcal{Q}_{6 n+1}^{\varepsilon}$. Arguing as in the proof of Proposition 3 , it is possible to compute the values of the mechanical fields for $n \geqslant N^{\varepsilon}$. Starting with (56)(57) and using Proposition 2, we see inductively that $\left(\omega_{6 n-3}^{\varepsilon}-\varepsilon\right)^{2}>\gamma_{2}$ and so

$\dot{\ell}_{2 n+1}^{\varepsilon}=\frac{1}{\varepsilon} \frac{\left(\omega_{6 n-3}^{\varepsilon}-\varepsilon\right)^{2}-\gamma_{2}}{\left(\omega_{6 n-3}^{\varepsilon}-\varepsilon\right)^{2}+\gamma_{2}}>0$

for $n \geqslant N^{\varepsilon}$.

We are now in position to apply [7, Lemma 3.2], setting

$$
X_{0}^{\varepsilon}:=\frac{\sqrt{2 \gamma_{2}}}{\left|\omega_{6 N^{\varepsilon}-3}^{\varepsilon}\right|}, X_{\mathrm{i}}^{\varepsilon}:=\frac{\sqrt{2 \gamma_{2}}}{\left|\omega_{6 \mathrm{~N}^{\varepsilon}-3+6 \mathrm{i}}^{\varepsilon}\right|}, \eta_{\varepsilon}:=\frac{\varepsilon}{\sqrt{2 \gamma_{2}}} .
$$

Indeed, it is possible to prove that $1 / X_{i+1}^{\varepsilon}=X_{i}^{\varepsilon}+2 \eta_{\varepsilon}$, so that by [7, Lemma 3.2] we obtain $X_{i}^{\varepsilon} \rightarrow \sqrt{1+\eta_{\varepsilon}^{2}}-\eta_{\varepsilon}$ as $i \rightarrow \infty$ (with oscillations). In particular, we find

$$
\begin{gathered}
\lim _{n \rightarrow \infty} \dot{\ell}_{2 n+1}^{\varepsilon}=\frac{1}{\sqrt{2 \gamma_{2}+\varepsilon^{2}}}=\dot{\ell}_{0}^{\varepsilon}, \\
\lim _{n \rightarrow \infty} \omega_{6 n+1}^{\varepsilon}=-\sqrt{2 \gamma_{2}+\varepsilon^{2}}=\omega_{1}^{\varepsilon} .
\end{gathered}
$$

Hence, the final stage presents the alternation of two phases: as before, the second shock wave restores the speed of propagation, which turns to be equal to $\dot{\ell}_{0}^{\varepsilon}$; the first shock wave changes the speed, which tends to $\dot{\ell}_{0}^{\varepsilon}$ as $T \rightarrow \infty$, with a behaviour similar to the case of a single discontinuity. Arguing as in [7, Proposition 3.3], 


$$
\ell_{1}=\quad \ell_{2} \quad \ell_{d}
$$

Fig. 7 Equivalent toughness in the case of a short defect (case II). Here, $\gamma_{1}=0.5, \gamma_{2}=2, \ell_{1}=1, \ell_{2}=1.5$.

it is possible to see that in the final stage the dynamic solutions converge as $\varepsilon \rightarrow 0$ to the propagation of speed $\frac{1}{\sqrt{2 \gamma_{2}}}$. In this section we have proven the following proposition.

Proposition 6 In case II, the dynamic solutions $\ell^{\varepsilon}$ converge, as the speed of loading $\varepsilon$ tends to zero, towards the following function:

$\tilde{\ell}(T)= \begin{cases}\frac{T}{\sqrt{2 \gamma_{2}}} & \text { if } 0 \leqslant T \leqslant \ell_{1} \sqrt{2 \gamma_{2}}, \\ \Phi^{-1}\left(\frac{T}{\sqrt{2 \gamma_{2}}}\right) & \text { if } \ell_{1} \sqrt{2 \gamma_{2}}<T \leqslant \ell_{d} \sqrt{2 \gamma_{2}}, \\ \frac{T}{\sqrt{2 \gamma_{2}}} & \text { if } T>\ell_{d} \sqrt{2 \gamma_{2}},\end{cases}$

where $\Phi^{-1}$ is the inverse of (73).

Notice that $\tilde{\ell}$ has a discontinuity at $\ell_{1} \sqrt{2 \gamma_{2}}$ and differs from the quasistatic solution (41) between $\ell_{1} \sqrt{2 \gamma_{2}}$ and $\ell_{d} \sqrt{2 \gamma_{2}}$ (see Figure 6).

\subsection{Equivalent quasistatic toughness}

In the previous sections, we compared the quasistatic and the dynamic solutions in the case of a defect and we showed that the quasistatic solution $\ell$ does not coincide with $\tilde{\ell}$, the limit of the dynamic ones as $\varepsilon \rightarrow 0$ (provided the defect is small, i.e., $\ell_{2}<\ell_{c}$ : see $\left.(32)\right)$. We refer to (41) and to (77) for the definition of $\ell$ and $\tilde{\ell}$. We assume $\ell_{2}<\ell_{c}$ (see $\left.(32)\right)$.

The differences between $\ell$ and $\tilde{\ell}$ raise a question on the effective toughness, which can be formulated in two different ways: (i) When a numerical analysis is performed, is it possible to obtain the dynamic (more representative) solution via a quasistatic calculation? (ii) If one measures experimentally the toughness of such a composite material, what will one get?

The aim of this section is to answer these questions by computing the equivalent quasistatic toughness $\widetilde{G}_{c}$ such that the quasistatic solution associated to $\widetilde{G}_{c}$ (assuming the criterion of energy conservation) coincides with $\tilde{\ell}$. This is indeed the natural procedure to obtain the effective quasistatic toughness of the composite material.

In the intervals $\left[0, \ell_{1}\right]$ and $\left(\ell_{d},+\infty\right)$ the equivalent toughness equals the original one, $N \gamma_{2}$ : indeed, $\tilde{\ell}$ coincides with the evolution corresponding to $N \gamma_{2}$ according to Griffith's classical theory. On the contrary, we shall modify the toughness in the interval $\left(\ell_{1}, \ell_{d}\right]$, where $\tilde{\ell}$ exhibits a particular behaviour.

In order to correct the length of the unstable crack propagation, the equivalent toughness can be determined using the criterion of energy conservation: since $\tilde{\ell}$ jumps from $\ell_{1}$ to $\ell_{2}$, we look for a new toughness such that $\mathcal{E}^{q}\left(T_{1}, \ell_{2}\right)=\mathcal{E}^{q}\left(T_{1}, \ell_{1}\right)$. This leads to define, using (29) and assuming the new toughness constant inside the defect,

$$
\widetilde{G}_{c}(x)=N \gamma_{2} \frac{\ell_{1}}{\ell_{2}} \quad \text { for } x \in\left(\ell_{1}, \ell_{2}\right]
$$

Notice that in this interval $\widetilde{G}_{c}$ is independent of the toughness $\gamma_{1}$ inside the defect.

Since $\dot{\tilde{\ell}}(T) \neq 0$ in $\left(\ell_{1} \sqrt{2 \gamma_{2}}, \ell_{d} \sqrt{2 \gamma_{2}}\right]$, Griffith's criterion (9) implies that $\widetilde{G}_{c}$ equals the energy release rate 
of the solution in this interval. Using (7), (72), and (73) we obtain

$$
\widetilde{G}_{c}(x)=N \gamma_{2} \frac{\Phi(x)^{2}}{x^{2}}
$$

if $x \in\left(\ell_{2}, \ell_{d}\right]$. As $\widetilde{G}_{c}\left(\ell_{d}\right)=N \gamma_{2}=G_{c}\left(\ell_{d}\right)$, the toughness is continuous at $\ell_{d}$. We have proven the following result.

Proposition 7 Given a defect $\left(\ell_{1}, \ell_{2}\right)$, we define the equivalent quasistatic toughness

$\widetilde{G}_{c}(x):= \begin{cases}N \gamma_{2} & \text { if } 0 \leqslant x \leqslant \ell_{1}, \\ N \gamma_{2} \frac{\ell_{1}}{\ell_{2}} & \text { if } \ell_{1}<x \leqslant \ell_{2}, \\ N \gamma_{2} \frac{\Phi(x)^{2}}{x^{2}} & \text { if } \ell_{2}<x \leqslant \ell_{d}, \\ N \gamma_{2} & \text { if } x>\ell_{d} .\end{cases}$

Then the dynamic solutions converge, as the speed of loading tends to zero, towards the evolution $\tilde{\ell}$ given by Griffith's quasistatic criterion, under the hypothesis of energy conservation, with toughness $\widetilde{G}_{c}$.

Figure 7 presents this equivalent toughness, which should be used for a quasistatic numerical analysis or which would be experimentally measured on such a composite. This equivalent toughness differs from the real one both inside and outside the defect, i.e., in the interval $\left(\ell_{1}, \ell_{d}\right)$. Inside the defect, the equivalent toughness is higher than the one of the material, whereas outside the defect the equivalent toughness is lower.

\subsection{Evolution of the kinetic energy}

In this section we study the evolution of the kinetic energy (see (16)). For the sake of simplicity, in what follows we assume that the second discontinuity $\ell_{2}$ is reached before the first backward shock wave $S_{1}^{\varepsilon-}$ is reflected on the axis $x=0$, i.e., we assume $T_{2}^{\varepsilon}<\tau_{1}^{\varepsilon}$ with $\tau_{1}^{\varepsilon}:=T_{1}^{\varepsilon}+\varepsilon \ell_{1}$ (see Section 1.3 for the definition of $\left.S_{i}^{\varepsilon \pm}\right)$. By (43) and (45) this condition reads

$\ell_{2}<\ell_{1} \frac{2\left(\sqrt{2 \gamma_{2}+\varepsilon^{2}}+\varepsilon\right)^{2}}{\left(\sqrt{2 \gamma_{2}+\varepsilon^{2}}+\varepsilon\right)^{2}+2 \gamma_{1}}$.

It is straightforward to check that, under this hypothesis, the same behaviour is repeated at every further interaction of the second shock waves: if $\left(\tau_{2 n+1}^{\varepsilon}, 0\right)$ denotes the point of reflection of the first shock wave $S_{2 n+1}^{\varepsilon-}$ on the axis $x=0$, then $T_{2 n+2}^{\varepsilon}<\tau_{2 n+1}^{\varepsilon}$. On the contrary, one has $T_{2 n+1}^{\varepsilon}>\tau_{2 n}^{\varepsilon}$, where $\left(\tau_{2 n}^{\varepsilon}, 0\right)$ corresponds to the reflection of the second shock wave $S_{2 n}^{\varepsilon-}$. This situation is pictured in Figure 8 .

More precisely, we assume the following condition on the lengths:

$\ell_{2} \leqslant \ell_{1} \frac{2 \gamma_{2}}{\gamma_{2}+\gamma_{1}}$ $\ell_{3}^{\varepsilon}=\ell_{4}^{\varepsilon}$

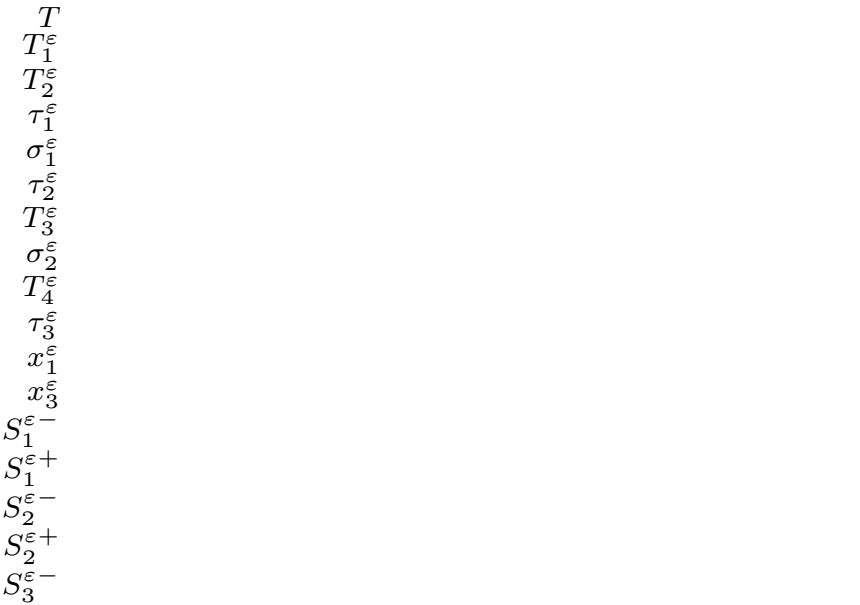

Fig. 8 Notation in the $(x, T)$-plane: the thick line represents the debonding, the thin ones are the shock waves.

which implies (79) for every $\varepsilon>0$. Moreover, (80) implies also (32), i.e., we are in case II. From the energetic point of view, (80) means that the kinetic energy is still increasing in the media when the second discontinuity is reached. Notice that in the opposite case $\left(\ell_{2}>\ell_{1} \frac{2 \gamma_{2}}{\gamma_{2}+\gamma_{1}}\right)$ we have $T_{2}^{\varepsilon}>\tau_{1}^{\varepsilon}$ for $\varepsilon$ small enough, but it may happen that $T_{2 n+2}^{\varepsilon}<\tau_{2 n+1}^{\varepsilon}$ for some index $n \geqslant 1$.

Recalling (13), (46)-(48), and (49)-(50), it is possible to compute the kinetic energy $\mathcal{K}^{\varepsilon}$ inside the media during the different stages of the evolution, and its limit as the speed of loading tends to zero. When the debonding meets the first discontinuity of the toughness we have $\mathcal{K}^{\varepsilon}\left(T_{1}^{\varepsilon}\right)=\frac{N L}{2} \varepsilon^{2} \ell_{1}$, so $\mathcal{K}^{\varepsilon}\left(T_{1}^{\varepsilon}\right) \rightarrow 0$ as $\varepsilon \rightarrow 0$. Afterwards, $\mathcal{K}^{\varepsilon}$ increases until the second discontinuity, reaching the value

$\mathcal{K}^{\varepsilon}\left(T_{2}^{\varepsilon}\right)=\frac{N L}{2}\left(\int_{0}^{x_{1}^{\varepsilon}}\left(\varepsilon v_{1}^{\varepsilon}(x)\right)^{2} \mathrm{~d} x+\int_{x_{1}^{\varepsilon}}^{\ell_{2}}\left(\varepsilon v_{2}^{\varepsilon}(x)\right)^{2} \mathrm{~d} x\right)$

where $x_{1}^{\varepsilon}=\ell_{1}-\frac{\ell_{2}-\ell_{1}}{\varepsilon \dot{\ell}_{1}^{\varepsilon}}$ is the coordinate of the intersection between the first backward shock wave $S_{1}^{\varepsilon-}$ and the line $T=T_{2}^{\varepsilon}$ (see Figure 8 ). A direct computation gives

$$
\widetilde{\mathcal{K}}_{2}:=\lim _{\varepsilon \rightarrow 0} \mathcal{K}^{\varepsilon}\left(T_{2}^{\varepsilon}\right)=\frac{N L}{2}\left(\ell_{2}-\ell_{1}\right)\left(\gamma_{2}-\gamma_{1}\right),
$$

which is interpreted as the value of some "limiting kinetic energy" at the length $\ell_{2}$.

In particular, when the debonding reaches the second discontinuity, the kinetic energy does not vanish. Indeed, during the unstable propagation, $\mathcal{K}^{\varepsilon}$ increases and then decreases; since $\ell_{2}<\ell_{c}, \mathcal{K}^{\varepsilon}$ is still positive when the front of debonding reaches the end of the defect. 
Remark 8 Until (80) holds, $\widetilde{\mathcal{K}}_{2}$ increases as $\ell_{2}$ does. If on the contrary $\ell_{2}>\ell_{1} \frac{2 \gamma_{2}}{\gamma_{2}+\gamma_{1}}$, repeating the arguments it is possible to show that $x_{1}^{\varepsilon}=\frac{\ell_{2}-\ell_{1}}{\varepsilon \dot{\ell}_{1}^{\varepsilon}}-\ell_{1}$ and $\widetilde{\mathcal{K}}_{2}=\frac{N L}{2}\left(\ell_{1} \gamma_{2}-\ell_{2} \gamma_{1}\right) \frac{\gamma_{2}-\gamma_{1}}{\gamma_{2}}$ (which is decreasing in $\left.\ell_{2}\right)$. Therefore, the map $\ell_{2} \mapsto \widetilde{\mathcal{K}}_{2}$ assumes its maximum value for $\ell_{2}=\ell_{1} \frac{2 \gamma_{2}}{\gamma_{2}+\gamma_{1}}$. In the sequel, we will always suppose that (80) holds.

As for the intermediate stage, following this procedure we can compute the kinetic energy $\mathcal{K}^{\varepsilon}\left(T_{2 n+2}^{\varepsilon}\right)$ at each point where the debonding restarts, i.e, at the points $\left(\ell_{2 n+2}^{\varepsilon}, T_{2 n+2}^{\varepsilon}\right)$ for $n=1, \ldots, N^{\varepsilon}-1$ (see $\left.(60)\right)$. Thanks to the hypothesis $(80), \mathcal{K}^{\varepsilon}\left(T_{2 n+2}^{\varepsilon}\right)$ depends just on the transverse velocity in the sectors $\mathcal{Q}_{6 n}^{\varepsilon}$ and $\mathcal{Q}_{6 n+2}^{\varepsilon}$. By (54)-(55) we have $v_{6 n}^{\varepsilon}=1$; comparing $\mathcal{Q}_{6 n+2}^{\varepsilon}$ with $\mathcal{Q}_{6 n}^{\varepsilon}$ and $\mathcal{Q}_{6 n+1}^{\varepsilon}$, by (21), (54)-(55), and (56)-(57) we have

$$
v_{6 n+2}^{\varepsilon}=\frac{\gamma_{2}-\gamma_{1}}{\varepsilon\left(\sqrt{2 \gamma_{2}+\varepsilon^{2}}+\varepsilon\right)}+1-n .
$$

Hence, for $n=1, \ldots, N^{\varepsilon}-1$

$$
\begin{aligned}
\mathcal{K}^{\varepsilon}\left(T_{2 n+2}^{\varepsilon}\right)=\frac{N L}{2} & \left(\int_{0}^{x_{2 n+1}^{\varepsilon}}\left(\varepsilon v_{6 n}^{\varepsilon}(x)\right)^{2} \mathrm{~d} x\right. \\
& \left.+\int_{x_{2 n+1}^{\varepsilon}}^{\ell_{2 n+2}^{\varepsilon}}\left(\varepsilon v_{6 n+2}^{\varepsilon}\right)^{2} \mathrm{~d} x\right),
\end{aligned}
$$

where $x_{2 n+1}^{\varepsilon} \in\left[0, \ell_{1}\right]$ is the coordinate of the intersection between the $(2 n+1)$-th backward shock wave $S_{2 n+1}^{\varepsilon-}$ and the line $T=T_{2 n+2}^{\varepsilon}$. A direct computation gives $x_{2 n+1}^{\varepsilon}=\ell_{2 n+2}^{\varepsilon}-\frac{\Delta^{\varepsilon}}{\varepsilon}$, with $\Delta^{\varepsilon}$ as in (61). Therefore,

$$
\begin{aligned}
\mathcal{K}^{\varepsilon}\left(T_{2 n+2}^{\varepsilon}\right)= & \frac{N L}{2} \varepsilon^{2}\left(\ell_{2 n+2}^{\varepsilon}-\frac{\Delta^{\varepsilon}}{\varepsilon}\right) \\
& +\frac{N L}{2} \frac{\Delta^{\varepsilon}}{\varepsilon}\left(\frac{\gamma_{2}-\gamma_{1}}{\sqrt{2 \gamma_{2}+\varepsilon^{2}}+\varepsilon}+\varepsilon(1-n)\right)^{2} .
\end{aligned}
$$

We can repeat the above arguments for each point of arrest, $\left(\ell_{2 n+1}^{\varepsilon}, T_{2 n+1}^{\varepsilon}\right)$ for $n=1, \ldots, N^{\varepsilon}-1$ (of course, $\left.\ell_{2 n+1}^{\varepsilon}=\ell_{2 n+2}^{\varepsilon}\right)$. Since $\mathcal{K}^{\varepsilon}\left(T_{2 n+1}^{\varepsilon}\right)$ depends just on the transverse velocity in the sectors $\mathcal{Q}_{6 n}^{\varepsilon}$ and $\mathcal{Q}_{6 n-1}^{\varepsilon}$, we compute

$$
v_{6 n-1}^{\varepsilon}=1-v_{6 n+2}^{\varepsilon}
$$

and we get

$$
\mathcal{K}^{\varepsilon}\left(T_{2 n+1}^{\varepsilon}\right)=\mathcal{K}^{\varepsilon}\left(T_{2 n+2}^{\varepsilon}\right)+O\left(\varepsilon^{2}\right)
$$

In order to describe the behaviour of the kinetic energy as $\varepsilon \rightarrow 0$, given $\ell \in\left(\ell_{2}, \ell_{d}\right)$ we set $n=\bar{n}^{\varepsilon}(\ell)$ in (82), where $\ell_{d}$ is defined in (74) and $\bar{n}^{\varepsilon}(\ell)$ is the integer such that $\ell_{2 \bar{n}^{\varepsilon}(\ell)+2}^{\varepsilon} \leq \ell<\ell_{2 \bar{n}^{\varepsilon}(\ell)+4}^{\varepsilon}($ see $(67)$ and $(68))$. Recalling (62) and (70), we obtain

$$
\begin{aligned}
\widetilde{\mathcal{K}}(\ell) & :=\lim _{\varepsilon \rightarrow 0} \mathcal{K}^{\varepsilon}\left(T_{2 \bar{n}^{\varepsilon}(\ell)+2}^{\varepsilon}\right)=\lim _{\varepsilon \rightarrow 0} \mathcal{K}^{\varepsilon}\left(T_{2 \bar{n}^{\varepsilon}(\ell)+3}^{\varepsilon}\right) \\
& =\frac{N L}{2} \frac{\ell_{2}-\ell_{1}}{\gamma_{2}-\gamma_{1}}\left(\gamma_{2}-\gamma_{1}-\gamma_{2} \phi(\ell)\right)^{2}
\end{aligned}
$$

where $\phi(\ell):=\ln \frac{\ell\left(\gamma_{2}-\gamma_{1}\right)-\left(\ell_{2}-\ell_{1}\right) \gamma_{2}}{\ell_{1} \gamma_{2}-\ell_{2} \gamma_{1}}$. Notice that the only sectors giving a contribution to the "limit" kinetic energy are the ones of the type $\mathcal{Q}_{6 n-1}^{\varepsilon}$ and $\mathcal{Q}_{6 n+2}^{\varepsilon}$.

As for the length $\ell_{2 N^{\varepsilon}}$ reached at the end of the intermediate stage (see $(58)$ ), with the notation introduced above we have $\bar{n}^{\varepsilon}\left(\ell_{2 N^{\varepsilon}}\right)=\varepsilon N^{\varepsilon}-\varepsilon$. Then, using (74) and (75) we get in the limit

$$
\widetilde{\mathcal{K}}\left(\ell_{d}\right)=\lim _{\varepsilon \rightarrow 0} \mathcal{K}^{\varepsilon}\left(T_{2 N^{\varepsilon}}^{\varepsilon}\right)=0,
$$

so the kinetic energy in the final stage vanishes as the speed of loading tends to zero. This suggests that the intermediate stage corresponds to a transformation of the kinetic energy created during the unstable propagation inside the defect. The quasistatic propagation starts again when all the kinetic energy has been transformed.

The function $\widetilde{\mathcal{K}}$ is naturally extended as

$$
\widetilde{\mathcal{K}}(\ell):= \begin{cases}0 & \text { if } 0 \leqslant \ell \leqslant \ell_{1}, \\ \frac{N L}{2}\left(\ell-\ell_{1}\right)\left(\gamma_{2}-\gamma_{1}\right) & \text { if } \ell_{1}<\ell \leqslant \ell_{2}, \\ \frac{N L}{2} \frac{\ell_{2}-\ell_{1}}{\gamma_{2}-\gamma_{1}}\left(\gamma_{2}-\gamma_{1}-\gamma_{2} \phi(\ell)\right)^{2} & \text { if } \ell_{2}<\ell \leqslant \ell_{d}, \\ 0 & \text { if } \ell>\ell_{d} .\end{cases}
$$

Hence, $\ell \mapsto \widetilde{\mathcal{K}}(\ell)$ is continuous and $\widetilde{\mathcal{K}}\left(\ell_{2}\right)=\widetilde{\mathcal{K}}_{2}$.

Actually, the kinetic energy $\mathcal{K}^{\varepsilon}(T)$ oscillates as $T$ varies between $T_{2}^{\varepsilon}$ and $T_{2 N^{\varepsilon}}^{\varepsilon}$ : this can be seen arguing as before and recalling the geometric structure of the sectors $\mathcal{Q}_{i}^{\varepsilon}$ (see Figure 8). In particular,

- $\mathcal{K}^{\varepsilon}$ is piecewise affine with $C^{1}$-discontinuities in the points $T_{m}^{\varepsilon}, \tau_{m}^{\varepsilon}$, and $\sigma_{m}^{\varepsilon}$, where the loads $\sigma_{m}^{\varepsilon}$ correspond to each intersection of the forward shock wave $S_{m}^{\varepsilon+}$ with the backward one $S_{m+1}^{\varepsilon-}\left(m=1, \ldots, 2 N^{\varepsilon}-\right.$ $2)$;

- the local minimum points are $\sigma_{m}^{\varepsilon}\left(m=1, \ldots, 2 N^{\varepsilon}-\right.$ 2 ); since in the associated sectors $\mathcal{Q}_{3 m}^{\varepsilon}$ and $\mathcal{Q}_{3 m+1}^{\varepsilon}$ the transverse velocity can assume only the values 0 or 1 , it turns out that $\mathcal{K}^{\varepsilon}\left(\sigma_{m}^{\varepsilon}\right)=O\left(\varepsilon^{2}\right)$;

- $\mathcal{K}^{\varepsilon}$ is increasing in $\left[0, \tau_{1}^{\varepsilon}\right]$, decreasing in every interval of the type $\left[\tau_{m}^{\varepsilon}, \sigma_{m}^{\varepsilon}\right]$, then increasing in $\left[\sigma_{m}^{\varepsilon}, \tau_{m+1}^{\varepsilon}\right]$ $\left(m=1, \ldots, 2 N^{\varepsilon}-2\right)$;

- the local maximum points are $\tau_{m}^{\varepsilon}\left(m=1, \ldots, 2 N^{\varepsilon}-\right.$ $2)$; arguing as before, it turns out that $\mathcal{K}^{\varepsilon}\left(\tau_{m}^{\varepsilon}\right)=$ $\mathcal{K}^{\varepsilon}\left(T_{m+1}^{\varepsilon}\right)+O\left(\varepsilon^{2}\right)$. 
Therefore, $\widetilde{\mathcal{K}}$ estimates the maximal oscillation of the kinetic energy when $\varepsilon \ll 1$.

More precisely, we consider the functions $\widetilde{\mathcal{K}}^{\varepsilon}(\ell):=$ $\mathcal{K}^{\varepsilon}\left(T^{\varepsilon}(\ell)\right)$, where $\ell \mapsto T^{\varepsilon}(\ell)$ denotes the inverse of $T \mapsto \ell^{\varepsilon}(T)$ (see $(71)$ for its expression in $\left[\ell_{2}, \ell_{2 N^{\varepsilon}}\right]$ ). The previous discussion shows that $\widetilde{\mathcal{K}}$ gives an estimate from above on the "limit" of $\widetilde{\mathcal{K}}^{\varepsilon}$, i.e., for every $\ell \geqslant 0$

$\widetilde{\mathcal{K}}(\ell) \geqslant \limsup _{\varepsilon \rightarrow 0} \widetilde{\mathcal{K}}^{\varepsilon}\left(l^{\varepsilon}\right) \quad$ for every possible $l^{\varepsilon} \rightarrow \ell$.

On the other hand, the last estimate is optimal, since for every $\ell \geqslant 0$

there exists $\bar{l}^{\varepsilon} \rightarrow \ell: \quad \widetilde{\mathcal{K}}(\ell)=\lim _{\varepsilon \rightarrow 0} \tilde{\mathcal{K}}^{\varepsilon}\left(\bar{l}^{\varepsilon}\right)$.

To prove (86) it suffices to take $\bar{l}^{\varepsilon}=\ell_{2 \bar{n}^{\varepsilon}(\ell)+2}^{\varepsilon}$ if $\ell \in$ $\left[\ell_{2}, \ell_{d}\right]$ and $\bar{l}^{\varepsilon}=\ell$ otherwise. Properties (85) and (86) imply the $\Gamma$-convergence of (the opposite of) the kinetic energy; we refer to [3] for the definition of this convergence.

Proposition 9 The functions $-\widetilde{\mathcal{K}}^{\varepsilon} \Gamma$-converge as $\varepsilon \rightarrow$ 0 to $-\widetilde{\mathcal{K}}$.

Notice that, if we consider the $\Gamma$-limit of $\widetilde{\mathcal{K}}^{\varepsilon}$ instead of the one of $-\widetilde{\mathcal{K}}^{\varepsilon}$, we get a trivial estimate: indeed, since $\mathcal{K}^{\varepsilon}$ is nonnegative and for every $\ell$ we have $\mathcal{K}^{\varepsilon}\left(\sigma_{2 \bar{n}^{\varepsilon}(\ell)+1}^{\varepsilon}\right) \rightarrow 0$, then the functions $\widetilde{\mathcal{K}}^{\varepsilon} \Gamma$-converge in $\left(\ell_{2}, \ell_{d}\right)$ to 0 as $\varepsilon \rightarrow 0$. This means that the "minimal oscillations" of the kinetic energy vanish between $\ell_{2}$ and $\ell_{d}$.

Thus, the function $\widetilde{\mathcal{K}}$ defined in (84) is interpreted as the limiting kinetic energy (in the sense of $\Gamma$-convergence) corresponding to the evolution $\tilde{\ell}$ introduced in (77); in other words, it is the kinetic energy associated to the equivalent toughness $\widetilde{G}_{c}$ (see (78)) under the assumption of energy conservation.

The oscillating behaviour of the kinetic energy has its counterpart in the potential energy $\mathcal{P}^{\varepsilon}$. Indeed, repeating the previous arguments it can be shown that the oscillations of $\mathcal{P}^{\varepsilon}$ are opposite to the ones of $\mathcal{K}^{\varepsilon}$ : the local minimum points of $\mathcal{P}^{\varepsilon}$ are (close to) the local maximum points of $\mathcal{K}^{\varepsilon}$, and viceversa. More precisely,

- $\mathcal{P}^{\varepsilon}$ is piecewise affine with $C^{1}$-discontinuities in the points $T_{m}^{\varepsilon}, \tau_{m}^{\varepsilon}$, and $\sigma_{m}^{\varepsilon}$ defined above;

- each of the points $\sigma_{m}^{\varepsilon}$ is a local maximum point $\left(m=1, \ldots, 2 N^{\varepsilon}-2\right)$;

- the local minimum points are of the type $T_{2 n}^{\varepsilon}, \tau_{2 n}^{\varepsilon}$ $\left(n=1, \ldots, N^{\varepsilon}-1\right)$.

Hence, in the limit as $\varepsilon \rightarrow 0$ we can determine for the potential energy an upper bound

$$
\begin{aligned}
\widetilde{\mathcal{P}}_{\max }(\ell) & :=\lim _{\varepsilon \rightarrow 0} \mathcal{P}^{\varepsilon}\left(\sigma_{2 \bar{n}^{\varepsilon}(\ell)+1}^{\varepsilon}\right)=\lim _{\varepsilon \rightarrow 0} \mathcal{P}^{\varepsilon}\left(\sigma_{2 \bar{n}^{\varepsilon}(\ell)+2}^{\varepsilon}\right) \\
& =N L\left(\frac{\ell_{2}-\ell_{1}}{\gamma_{2}-\gamma_{1}}\left(\gamma_{1}+\gamma_{2} \phi(\ell)\right)^{2}-\frac{\ell_{2}-\ell_{1}}{\gamma_{2}-\gamma_{1}} \gamma_{2}^{2}+\ell \gamma_{2}\right)
\end{aligned}
$$

and a lower bound

$$
\begin{gathered}
\widetilde{\mathcal{P}}_{\min }(\ell):=\lim _{\varepsilon \rightarrow 0} \mathcal{P}^{\varepsilon}\left(T_{2 \bar{n}^{\varepsilon}(\ell)+2}^{\varepsilon}\right)=\lim _{\varepsilon \rightarrow 0} \mathcal{P}^{\varepsilon}\left(T_{2 \bar{n}^{\varepsilon}(\ell)+3}^{\varepsilon}\right) \\
=N L\left(\frac{1}{2} \frac{\ell_{2}-\ell_{1}}{\gamma_{2}-\gamma_{1}}\left(\gamma_{1}+\gamma_{2}+\gamma_{2} \phi(\ell)\right)^{2}\right. \\
\left.-2 \frac{\ell_{2}-\ell_{1}}{\gamma_{2}-\gamma_{1}} \gamma_{2}^{2}+\ell \gamma_{2}\right) .
\end{gathered}
$$

We have also

$$
\widetilde{\mathcal{P}}_{\min }(\ell)=\lim _{\varepsilon \rightarrow 0} \mathcal{P}^{\varepsilon}\left(\tau_{2 \bar{n}^{\varepsilon}(\ell)+1}^{\varepsilon}\right)=\lim _{\varepsilon \rightarrow 0} \mathcal{P}^{\varepsilon}\left(\tau_{2 \bar{n}^{\varepsilon}(\ell)+2}^{\varepsilon}\right) .
$$

As before, this bounds are optimal: indeed, if we define $\widetilde{\mathcal{P}}^{\varepsilon}(\ell):=\mathcal{P}^{\varepsilon}\left(T^{\varepsilon}(\ell)\right)$ for $\ell_{2}<\ell<\ell_{d}$, the functions $\widetilde{\mathcal{P}}^{\varepsilon}$ $\Gamma$-converge in $\left(\ell_{2}, \ell_{d}\right)$ to $\widetilde{\mathcal{P}}_{\text {min }}$, while their opposites $\Gamma$ converge to $-\widetilde{\mathcal{P}}_{\max }$. Both these limits are nondecreasing functions of $\ell$, since the load is monotonic.

Finally, notice that

$$
\widetilde{\mathcal{P}}_{\max }(\ell)-\widetilde{\mathcal{P}}_{\min }(\ell)=\widetilde{\mathcal{K}}(\ell) .
$$

The mechanical interpretation is that the oscillations correspond to transformations of kinetic energy in potential energy, and viceversa. At each step the amount of kinetic energy is lower, so the process finishes when the kinetic energy has been completely relaxed; afterwards, the system switches to a quasistatic behaviour, where the kinetic energy is negligible.

\subsection{Conclusion}

In this section we have compared evolutions of quasistatic and dynamic type in the debonding of a thin film with one defect (of toughness lower than the one of the material). The quasistatic propagation is determined using the principle of energy conservation.

Two cases are possible. In the first, where the defect is long, the quasistatic solution coincides with the limit of the dynamic ones as the loading speed tends to zero. The evolution contains an unstable stage and an arrest inside the defect, followed by a slow propagation and a second arrest at the end of the defect. This case in fact corresponds to a combination of two discontinuities, the first one without any influence on the second, due to the large length of the defect: indeed, the kinetic energy created during the unstable stage vanishes when the debonding reaches the second discontinuity.

In the second case, where the defect is small enough, the dynamic solutions do not converge to the quasistatic one. Indeed, a complex behaviour (though, limited in time) emerges after the second discontinuity: this is due to the presence of residual kinetic energy when the 
debonding reaches the second discontinuity. The equivalent quasistatic toughness (78) allows one to find the limit of the dynamic response using only quasistatic criteria.

This analysis shows two different behaviours of the kinetic energy. In the part with the lowest toughness there is an increase in the kinetic energy during the unstable propagation. On the contrary, after the defect the kinetic energy decreases and is transformed into potential and surface energy, while the loading increases.

\section{The case of an infinite number of defects}

In the previous sections we have studied the role of the kinetic energy in the debonding of a thin film, in the cases of one discontinuity of the toughness and one defect (two discontinuities). A specific behaviour of the kinetic energy has been highlighted: it is created when the debonding propagates inside a zone of lower touhgness and vanishes in a logarithmic fashion in the zones of higher toughness. We consider now the case of infinite defects with vanishing size: the quasistatic solution can be determined analytically (under the assumption of energy conservation), while the dynamic solutions are studied numerically, due to the difficulties of computation. The simulations show different possible qualitative behaviours for the dynamic solutions, depending on the density of the material with the lowest toughness.

\subsection{Quasistatic solution}

The following periodic repartition of the toughness is considered,

$G_{c}(x)= \begin{cases}\gamma_{1} N & \text { if } x \in(i d,(i+\theta) d) \text { for } i \in \mathbb{N}, \\ \gamma_{2} N & \text { if } x \in((i+\theta) d,(i+1) d) \text { for } i \in \mathbb{N}\end{cases}$

where $0<\gamma_{1}<\gamma_{2}$ again, $\theta \in(0,1)$ is the density of the lowest toughness $\gamma_{1}, d$ is the period, and $(1-\theta) d$ is the length of each defect. Even if all our arguments work without assumptions on $\theta$, in the following simulations we consider in particular the case where $\theta$ is close to 1 : hence we model a material of toughness $\gamma_{1}$ with small inclusions (defects) of higher toughness $\gamma_{2}$.

We are interested in the limit as the number of defects tends to infinity (homogenized material), so the length $d$ vanishes. We sketch here the algorithm to determine the quasistatic solution (under the principle of energy conservation) and its limit as $d \rightarrow 0$; a detailed analysis can be done using the arguments of Section 2.2. As shown before, in the quasistatic framework
Fig. 9 Limit of the quasistatic evolution for $d \rightarrow 0$ (continuous line). The dashed lines have slope $\sqrt{2 \gamma_{2}}$ and $2 \bar{\gamma} / \sqrt{2 \gamma_{2}}$ and correspond to quasistatic evolutions for homogeneous materials of toughness $\gamma_{2}$ and $\bar{\gamma}^{2} / \gamma_{2}$, respectively.

the debonding front may jump, be constant, or evolve with the two possible speeds $1 / \sqrt{2 \gamma_{1}}$ and $1 / \sqrt{2 \gamma_{2}}$, according to the toughness of the zone where the front is.

More precisely, let us consider a discontinuity of the type $\tilde{\ell}_{i}:=i d$ (decreasing toughness), reached with speed $1 / \sqrt{2 \gamma_{2}}$ and load $\widetilde{T}_{i}:=\sqrt{2 \gamma_{2}} i d$. From here the length jumps to the (minimal) point $\ell^{+}(i)>\tilde{\ell}_{i}$ such that $\mathcal{E}^{q}\left(\widetilde{T}_{i}, \tilde{\ell}_{i}\right)=\mathcal{E}^{q}\left(\widetilde{T}_{i}, \ell^{+}(i)\right)$, according to the criterion of energy conservation presented before (see (8) for the definition of the quasistatic energy $\left.\mathcal{E}^{q}\right)$. Afterwards,

- if $\ell^{+}(i)$ is in a zone of toughness $\gamma_{2}$, the debonding front remains constant until the loading level $\sqrt{2 \gamma_{2}} \ell^{+}(i)$ is reached, then the debonding can restart with speed $1 / \sqrt{2 \gamma_{2}}$;

- otherwise, if $\ell^{+}(i)$ is in a zone of toughness $\gamma_{1}$, the debonding front remains constant until the loading level $\sqrt{2 \gamma_{1}} \ell^{+}(i)$ is reached, then the debonding can restart with speed $1 / \sqrt{2 \gamma_{1}}$; at the following discontinuity (increasing toughness), the debonding front remains constant until it reaches a loading level so high that it can restart with speed $1 / \sqrt{2 \gamma_{2}}$, again.

In both cases, the debonding meets a discontinuity with decreasing toughness, so it jumps as described above; and so on.

As $d \rightarrow 0$, the transition between $\ell^{+}(i)$ and the new jump point tends (in both cases) to a constant evolution; therefore, the limit propagation as $d \rightarrow 0$ (homogenized case) is a staircase function, composed just of jumps and stops. The mechanical interpretation is that the systems stores potential energy during the arrest phase, until this energy is sufficient to create an unstable propagation of the crack. Moreover, by a direct 
computation it turns out that

$$
\lim _{d \rightarrow 0} \frac{\ell^{+}(i)}{\widetilde{T}_{i}}=\frac{\sqrt{2 \gamma_{2}}}{2 \bar{\gamma}},
$$

where $\bar{\gamma}:=\theta \gamma_{1}+(1-\theta) \gamma_{2}$ is the mean toughness. Hence, the bounds for the limit staircase evolution (in the $(x, T)$-plane) are two lines of respective slopes $\sqrt{2 \gamma_{2}}$ and $\frac{2 \bar{\gamma}}{\sqrt{2 \gamma_{2}}}$. The quasistatic evolution is pictured in Figure 9 .

Notice that $\frac{2 \bar{\gamma}}{\sqrt{2 \gamma_{2}}}<\sqrt{2 \bar{\gamma}}$ : this means that the naïve homogenization (using the mean toughness $\bar{\gamma}$ ) leads to underestimate the propagation of the debonding found under the assumption of energy conservation. In addition, for $\theta$ sufficiently close to 1 (the case of a material with small inclusions of higher toughness) we have $\frac{2 \bar{\gamma}}{\sqrt{2 \gamma_{2}}}<\sqrt{2 \gamma_{1}}$ : therefore, in a homogenized material with low density of the highest toughness, the expected length of propagation during the fast growth is greater than the one corresponding to the pure material with the lowest toughness. This non-intuitive result will be enforced by the dynamic analysis.

\subsection{Dynamic solutions}

In the previous section, we analyzed the quasistatic solution in the case of infinite discontinuities, showing a structure of staircase evolution. We consider here the dynamic solutions; however, due to the large amount of computations needed, we provide only some numerical results. Since the method presented in Section 1.3 would lead to a number of quantities impossible to manage, we use an alternative approach based on the properties of the wave equation, which allows us to deal with a great number of discontinuities (we perform the method in a lattice of $10^{5}$ defects). The drawback is that now the kinetic energy cannot be easily computed: this makes the interpretation more difficult.

\subsubsection{Exact recursive scheme for the wave equation}

For every $\varepsilon>0$, let us consider the transverse component $w^{\varepsilon}$ of the displacement field with its derivatives $\omega^{\varepsilon}$ and $v^{\varepsilon}$ (see (2) and (13)). By the classical properties of the wave equation (17), we can find two functions $f^{\varepsilon}, g^{\varepsilon}: \mathbb{R} \rightarrow \mathbb{R}$ such that

$$
w^{\varepsilon}(x, T)=f^{\varepsilon}(T-\varepsilon x)+g^{\varepsilon}(T+\varepsilon x)
$$

for every $(x, T) \in(0,+\infty)^{2}$. Passing to the derivatives, we get $\omega^{\varepsilon}=\varepsilon\left(\left(g^{\varepsilon}\right)^{\prime}-\left(f^{\varepsilon}\right)^{\prime}\right)$ and $v^{\varepsilon}=\left(f^{\varepsilon}\right)^{\prime}+\left(g^{\varepsilon}\right)^{\prime}$. Moreover, using $(20)$ we can assume $\left(g^{\varepsilon}\right)^{\prime}=1-\left(f^{\varepsilon}\right)^{\prime}$ everywhere in $\mathbb{R}$, so we can express the dynamic fields $\omega^{\varepsilon}$ and $v^{\varepsilon}$ by $\psi^{\varepsilon}:=\left(f^{\varepsilon}\right)^{\prime}$.
We now determine some conditions on the function $T \mapsto \ell^{\varepsilon}(T)$ using equations (22), (24), and (25). By (22) we obtain

$\psi^{\varepsilon}\left(T+\varepsilon \ell^{\varepsilon}(T)\right)=1+\frac{1-\varepsilon \dot{\ell}^{\varepsilon}(T)}{1+\varepsilon \dot{\ell}^{\varepsilon}(T)} \psi^{\varepsilon}\left(T-\varepsilon \ell^{\varepsilon}(T)\right)$.

Furthermore, by (24), (25), and (90), repeating the arguments of the proof of Proposition 2 we see that

$\dot{\ell}^{\varepsilon}(T)=\frac{1}{\varepsilon} \frac{\left(2 N \varepsilon^{2} \psi^{\varepsilon}\left(T-\varepsilon \ell^{\varepsilon}(T)\right)^{2}-G_{c}\left(\ell^{\varepsilon}(T)\right)\right)^{+}}{2 N \varepsilon^{2} \psi^{\varepsilon}\left(T-\varepsilon \ell^{\varepsilon}(T)\right)^{2}+G_{c}\left(\ell^{\varepsilon}(T)\right)}$,

where $(\cdot)^{+}$stands for the positive part. The previous equations are understood to hold where the time derivative is defined.

Using (90) and (91), it is possible to perform an exact scheme for the solution of the dynamic problem even with a large number of discontinuities of the toughness. Recalling the structure of the solution seen in the previous sections, the function $\ell^{\varepsilon}$ is piecewise affine, so one has to determine the sequence $\left(\ell_{i}^{\varepsilon}, T_{i}^{\varepsilon}\right)$ of points where the speed changes, due either to the effect of discontinuities or to the interaction with the shock waves; the number of interactions between discontinuities depends in fact on the length of the defects (see e.g. Section 2.3.2 in the case of one defect, with no interactions before the second discontinuity). As previously, the speed between $T_{i}^{\varepsilon}$ and $T_{i+1}^{\varepsilon}$ is denoted by $\dot{\ell}_{i}^{\varepsilon}$.

Since the speed is piecewise constant and (91) holds, the functions $\psi^{\varepsilon}$ defined above are piecewise constant, too, with discontinuities in the points $T_{i}^{\varepsilon} \pm \varepsilon \ell_{i}^{\varepsilon}$, which correspond to the reflections of the shock waves on the axis $x=0$. By definition of point of reflection, if at the load $T_{i}^{\varepsilon}$ there are $M_{i}$ waves acting in the film, then $M_{i} \leqslant i$ and

$T_{i}^{\varepsilon}-\varepsilon \ell_{i}^{\varepsilon}=T_{i-M_{i}}^{\varepsilon}+\varepsilon \ell_{i-M_{i}}^{\varepsilon}$,

so it is sufficient to consider only the sequence $T_{i}^{\varepsilon}+\varepsilon \ell_{i}^{\varepsilon}$. We set $\left(\ell_{0}^{\varepsilon}, T_{0}^{\varepsilon}\right):=(0,0)$.

In what follows, $\psi_{i}^{\varepsilon}$ denotes the value of $\psi^{\varepsilon}$ between $T_{i}^{\varepsilon}+\varepsilon \ell_{i}^{\varepsilon}$ and $T_{i+1}^{\varepsilon}+\varepsilon \ell_{i+1}^{\varepsilon}$. Recalling (92), it is possible to translate (90) and (91) into the following recursive relations:

$\psi_{i}^{\varepsilon}=1+\frac{1-\varepsilon \dot{\ell}_{i}^{\varepsilon}}{1+\varepsilon \dot{\ell}_{i}^{\varepsilon}} \psi_{i-M_{i}}^{\varepsilon}$,

$\dot{\ell}_{i}^{\varepsilon}=\frac{1}{\varepsilon} \frac{\left(2 N \varepsilon^{2}\left(\psi_{i-M_{i}}^{\varepsilon}\right)^{2}-G_{c}\left(\ell_{i}\right)\right)^{+}}{2 N \varepsilon^{2}\left(\psi_{i-M_{i}}^{\varepsilon}\right)^{2}+G_{c}\left(\ell_{i}\right)}$,

where $M_{i}$ is the number of waves acting in the film at the load $T_{i}^{\varepsilon}$. Notice that the debonding proceeds with 
Fig. 10 Dynamic solutions in the case of many defects. Here, $\gamma_{1}=0.5, \gamma_{2}=2, \varepsilon=0.001, d=0.001$.

the speed prescribed by (94) only until a new discontinuity is met; then the number $M_{i}$ must be incremented.

More precisely, we describe here the inductive computation of $\ell_{i}^{\varepsilon}, T_{i}^{\varepsilon}, \dot{\ell}_{i}^{\varepsilon}$, and $\psi_{i}^{\varepsilon}$. The values for $i=0$ are found as mentioned in Section 2.3.1: we have $\left(\ell_{0}^{\varepsilon}, T_{0}^{\varepsilon}\right)=$ $(0,0)$ and $\dot{\ell}_{0}^{\varepsilon}=1 / \sqrt{2 G_{c}(0)+\varepsilon^{2}}$, while a direct computation shows that

$$
\psi_{0}^{\varepsilon}=\frac{\sqrt{2 G_{c}(0)+\varepsilon^{2}}+\varepsilon}{2 \varepsilon} .
$$

We set $M_{0}:=0$.

Let us assume now the values of $\ell_{i-1}^{\varepsilon}, T_{i-1}^{\varepsilon}, \dot{\ell}_{i-1}^{\varepsilon}$, and $\psi_{i-1}^{\varepsilon}$ to be known; suppose that $M_{i-1}$ waves are acting in the film. Hence, we compute the intersection $\left(\ell^{*}, T^{*}\right)$ between the front of debonding (a line starting at $\left(\ell_{i-1}^{\varepsilon}, T_{i-1}^{\varepsilon}\right)$ with slope $1 / \dot{\ell}_{i-1}^{\varepsilon}$, possibly parallel to the axis $T=0$ if $\left.\dot{\ell}_{i-1}^{\varepsilon}=0\right)$ and the shock wave starting at $\left(0, T_{i-M_{i-1}}^{\varepsilon}+\varepsilon \ell_{i-M_{i-1}}^{\varepsilon}\right)$ with slope $\varepsilon$. Then

- if no discontinuities of the toughness are present in the interval $\left(\ell_{i-1}^{\varepsilon}, \ell^{*}\right)$, we set $\left(\ell_{i}^{\varepsilon}, T_{i}^{\varepsilon}\right):=\left(\ell^{*}, T^{*}\right)$ and $M_{i}:=M_{i-1}$;

- if a discontinuity of the toughness is met in $\bar{\ell} \in$ $\left(\ell_{i-1}^{\varepsilon}, \ell^{*}\right)$ for some load $\bar{T}$, we set $\left(\ell_{i}^{\varepsilon}, T_{i}^{\varepsilon}\right):=(\bar{\ell}, \bar{T})$ and $M_{i}:=M_{i-1}+1$.

Finally, in both cases we compute $\dot{\ell}_{i}^{\varepsilon}$ and $\psi_{i}^{\varepsilon}$ using (93) and (94). Notice that these values take into account the possible presence of a new shock wave and the possible change in toughness.

\subsubsection{Analysis of the dynamic solutions in dependence} on $\theta$

The method described in the previous section permits us to perform the numerical analysis of a material with many defects. We study in particular how the qualitative aspect of the limit dynamic evolution (as $\varepsilon \rightarrow 0$ and $d \rightarrow 0$ ) is influenced by the choice of the parameter $\theta$, that is, the density of the lowest toughness in the defects' distribution given in (89). In the following pictures we fix $\gamma_{1}=0.5, \gamma_{2}=2, \varepsilon=0.001$, and $d=0.001$, and present the dynamic evolutions obtained for different values of $\theta$.

We compare the evolution of the debonding with periodic defects' distribution (89) for $d$ small, with the solution for a homogeneous film of toughness $\bar{\gamma}:=\theta \gamma_{1}+$ $(1-\theta) \gamma_{2}$. The two answers are different, since an effective toughness different from $\bar{\gamma}$ must be considered for the study of the homogenized material.

Figure 10 presents the dynamic evolutions obtained for different values of the densities of the lowest toughness. The simulation shows that the behaviour of the debonding depends strongly on $\theta$.

For $\theta$ close to 1 , the debonding curves show a wave trend. When $\theta$ is close to $1 / 2$, they approach a straight line, whose slope is greater than $\sqrt{2 \bar{\gamma}}$, i.e., greater than the slope of the line corresponding to the mean toughness $\bar{\gamma}$. Therefore, in the limit as $\varepsilon \rightarrow 0$ the debonding speed is lower than the one prescribed by the pure quasistatic approach in a homogeneous setting.

However, when $\theta$ is very close to 1 (i.e., when the film is quasi homogeneous and $\bar{\gamma}$ is close to $\gamma_{1}$ ), a dif- 


\section{$\frac{2 \gamma_{1}}{\sqrt{2 \gamma_{2}}}$}

Fig. 11 Dynamic solutions at a different scale. Here, $\gamma_{1}=0.5, \gamma_{2}=2, \varepsilon=0.001, d=0.001$.

ferent phenomenon emerges: the curve approaches a staircase function in the $(x, T)$-plane, bounded by two lines of respective slopes close to $\sqrt{2 \gamma_{2}}$ and $\frac{2 \bar{\gamma}}{\sqrt{2 \gamma_{2}}}$. This turns out to be the same trend predicted in the quasistatic context by the energetic criterion presented in Section 3.1. Thus, when the density of the lowest toughness is close to 1 , the properties of the debonding evolution could be studied through the quasistatic approach based on energy conservation, introduced above.

Consequently, the behaviour of a homogeneous material is completely different from the heterogeneous case even with density of the lowest toughness tending towards 1 . In addition, performing a classical quasistatic analysis with an average value of the toughness would lead to underestimate the length of debonding.

The case of $\theta$ close to 1 recalls the competition of the two phenomena, described above in the case of a single defect: the kinetic energy increases as the debonding passes from a zone of high toughness to a one with low toughness; afterwards, the created energy is relaxed out of the defect. In other words, when the debonding stops at the interface between low and high toughness, a part of the accumulated energy is transformed into kinetic energy; then, the debonding passes through a large number of defects (recall that in the simulations presented here, the size $d$ of the periodic pattern is 0.001). The kinetic energy is transformed (quasi) totally into Griffith's surface energy during the fast propagation. On the contrary, when the density of the highest toughness decreases, the kinetic energy is not transformed but accumulated, leading to a complex behaviour that cannot be captured by a quasistatic criterion.
Two scales are here involved: in the small scale of the defects, every single interface modifies the speed of debonding, so arrests and fast propagations occur, and the energy is transformed; yet, in the macroscopic scale a global behaviour is showed. Indeed, if $\theta$ is close to 1 , the curves show a macroscopic phase of arrest and a macroscopic phase of fast propagation, bounded by the two lines characterizing the quasistatic evolution: so, the system seems to present a characteristic length, increasing as the debonding evolves. On the other hand, the macroscopic behaviour is repeated in a self-similar fashion around the initial instant $(\ell=0, T=0)$ : this is shown in Figure 11, which is a zoom of the previous one close to the initial point. Unfortunately, the self-similarity seems too complex to be detemined by analytical arguments, which would require the solution of a nonconvex dynamic homogeneization problem.

The dependence of the solution on $\theta$ can be understood through a limit analysis. Repeating the numerical simulations for several small values of $\varepsilon$ and $d$, we find similar results in all the three cases $\varepsilon \sim d$, $\varepsilon \ll d$, and $\varepsilon \gg d$, i.e., when $\varepsilon$ tends to zero together with, before than, or later than $d$, respectively. For instance, we present in Figures 12, 13, 14, and 15 some curves with different values of the two parameters. Since the qualitative behaviour looks the same, this suggests that the two limits as $\varepsilon \rightarrow 0$ and $d \rightarrow 0$ commute. Of course, this does not mean that we expect pointwise convergence of the solutions: in fact, as the two parameters vary, the positions of the jump points change, so we see a translation of the phases of propagation and arrest.

Finally, the choice of the defects' distribution seems to have no influence on the possible shapes assumed 


$$
\begin{aligned}
& T \\
& \begin{array}{ll}
d & =0.1 \\
d & =0.01 \\
d & =0.001 \\
d & =0.0001
\end{array}
\end{aligned}
$$

$$
\frac{2 \gamma_{1}}{\sqrt{2 \gamma_{2}}}
$$

Fig. 12 Limit solution when $\varepsilon$ tends to 0 faster than $d$. Here, $\gamma_{1}=0.5, \gamma_{2}=2, d=0.01, \theta=0.97$.

$$
\frac{2 \gamma_{1}}{\sqrt{2 \gamma_{2}}}
$$

Fig. 13 Limit solution when $d$ tends to 0 faster than $\varepsilon$. Here, $\gamma_{1}=0.5, \gamma_{2}=2, \varepsilon=0.01, \theta=0.97$.

by the evolutionary curves, which are piloted only by $\theta$. In order to show this property, we provide in Figure 16 the result of a simulation where the defects are distributed randomly, in such a way that the mean density of the lowest toughness is $\theta$. The answer of the debonding for a random repartition of defects presents the same characteristics seen in the periodic framework: the modification of the position of the defects gives only a quantitative modification (curve translation).

\subsection{Conclusion}

In this section we have studied a material with many defects, whose number diverges and whose distribution could be periodic or random. In particular we have considered the case where the density of the highest toughness tends to zero. The limit behaviour is different from the case of a homogeneous material and highlights the effects of the kinetic energy as in the case of a single defect, discussed in Section 2. The quasistatic problem has been treated with analytical methods, while in the dynamic context only some numerical simulations have been performed. 
$T$

$$
\begin{aligned}
& d=0.1 \\
& d=0.01 \\
& d=0.001 \\
& d=0.0001
\end{aligned}
$$

Fig. 14 Limit solution when $\varepsilon$ tends to 0 faster than $d$. Here, $\gamma_{1}=0.5, \gamma_{2}=2, d=0.01, \theta=0.99999$.

$$
\frac{2 \gamma_{1}}{\sqrt{2 \gamma_{2}}}
$$

Fig. 15 Limit solution when $d$ tends to 0 faster than $\varepsilon$. Here, $\gamma_{1}=0.5, \gamma_{2}=2, \varepsilon=0.01, \theta=0.99999$.

In the quasistatic model (under the assumption of energy conservation), the solutions are staircase functions in the space/time plane. The dynamic solutions depend strongly on the density of the lowest toughness, while the choice of the defects' distribution (periodic or random) plays a minor role. In addition, the limit is qualitatively the same if $\varepsilon \ll d$ or if viceversa $d \ll \varepsilon$.

If the density of the lowest toughness is close to one, the dynamic solutions converge to a staircase evolution, with the same properties of the one found in the quasistatic framework: indeed, during the fast propagations the debonding passes through many defects. As this density decreases, the curves in the space/time plane seem smoother, showing a wave trend; if the density is close to $1 / 2$, their shape approaches a line, whose slope is however different from the one corresponding to the mean toughness.

This behaviour reflects the influence of the kinetic energy on the dynamics. Indeed, we see here the competition of two opposite phenomena: the creation of kinetic energy as the toughness decreases and the relaxation observed in the case of a single defect. 
Fig. 16 Dynamic solutions for a random distribution of defects of length $d(1-\theta)$. Here, $\gamma_{1}=0.5, \gamma_{2}=2, \varepsilon=0.001, d=0.001$.

\section{Concluding remarks}

The present study clearly proves that toughness heterogeneities induce inertial effects which cannot be negligible even in the case of a quasi-static loading. The solution is necessarily dynamic and the kinetic energy plays an important role in the propagation of cracks. However, the present study suggests also that there exists a limit effective behaviour when the speed of loading and the size of the heterogeneities go to zero. Beyond the somewhat chaotic motion of waves which propagate inside the body, it seems that an order takes place at the macroscopic level. What is this effective dynamic debonding law? Which macroscopic quantities give rise to the computed macroscopic behaviour? Even in our very simple case of a film debonding, we have been unable to answer to these fundamental questions. It is a very exciting challenge to advance in the understanding of this nonlinear dynamic homogenization problem and future theoretical works will be devoted to this task.

Acknowledgements This research was supported by the Fédération Francilienne de Mécanique through the project "La ténacité effective dynamique d'un composite" in collaboration with the Institut d'Alembert (Université Paris VI) and the Laboratoire de Mécanique des Structures Industrielles Durables (EDF R\&D).

Afterwards, G.L. was partly granted by the Deutsche Forschungsgemeinschaft through the Project SCHL 1706/2-1.
Table 1 Notation

\begin{tabular}{|c|c|}
\hline$x_{1}$ & coordinate on the film \\
\hline$W$ & opening displacement \\
\hline$L$ & characteristic length \\
\hline$N$ & tension \\
\hline$x$ & rescaled coordinate on the film \\
\hline$T$ & rescaled displacement \\
\hline$t$ & time \\
\hline$\varepsilon$ & loading speed (adimensional) \\
\hline$c$ & velocity of transversal waves \\
\hline$\rho$ & density of the film \\
\hline $\mathcal{E}^{q}$ & quasistatic total energy \\
\hline $\mathcal{K}^{\varepsilon}$ & kinetic energy \\
\hline $\mathcal{P}, \mathcal{P}^{\varepsilon}$ & potential energy \\
\hline $\mathcal{S}, \mathcal{S}^{\varepsilon}$ & surface energy \\
\hline$G$ & quasistatic energy release rate \\
\hline$G^{\varepsilon}$ & dynamic energy release rate \\
\hline$G_{c}$ & material toughness \\
\hline$\widetilde{G}_{c}$ & effective toughness \\
\hline$N \gamma_{1}, N \gamma_{2}$ & values for material toughness \\
\hline$N \bar{\gamma}$ & mean toughness \\
\hline$\ell_{1}, \ell_{2}$ & toughness discontinuities \\
\hline $\mathbf{u}$ & displacement field \\
\hline$u$ & first component of $\mathbf{u}$ \\
\hline$w, w^{\varepsilon}$ & second component of $\mathbf{u}$ \\
\hline$\omega^{\varepsilon}$ & infinitesimal rotation \\
\hline$v^{\varepsilon}$ & transverse velocity \\
\hline $\mathcal{Q}$ & $(x, T)$-plane \\
\hline $\mathcal{Q}_{0}, \mathcal{Q}_{0}^{\varepsilon}, \mathcal{Q}_{i}^{\varepsilon}$ & sectors in $\mathcal{Q}$ \\
\hline$\Gamma^{\varepsilon}$ & debonding front \\
\hline$S^{\varepsilon}, S^{\varepsilon \pm}$ & shock waves \\
\hline$\ell$ & quasistatic debonding \\
\hline$\ell^{\varepsilon}$ & dynamic debonding \\
\hline$\ell_{c}$ & final point after jump (single discontinuity) \\
\hline$\ell_{b_{\sim}}$ & final point after jump (defect) \\
\hline$\tilde{\ell}$ & limit of $\ell^{\varepsilon}$ \\
\hline$\ell_{d}$ & point where $\tilde{\ell}$ joins $\ell$ \\
\hline$\sim \widetilde{\mathcal{K}}$ & optimal bound for $\mathcal{K}^{\varepsilon}$ \\
\hline$\widetilde{\mathcal{P}}_{\min }, \widetilde{\mathcal{P}}_{\max }$ & optimal bounds for $\mathcal{P}^{\varepsilon}$ \\
\hline
\end{tabular}




\section{References}

1. J. D. Achenbach, Wave Propagation in Elastic Solids. North-Holland, Amsterdam (1973)

2. B. Bourdin, G. A. Francfort, and J.-J. Marigo, The variational approach to fracture, J. Elasticity, 91, 5-148 (2008)

3. A. Braides, $\Gamma$-convergence for beginners. Oxford University Press, Oxford (2002)

4. H. D. Bui, Mécanique de la Rupture Fragile. Masson, Paris (1978)

5. Y. L. Cui, Dynamic matrix cracking in fiber reinforced ceramics, J. Mech. Phys. Solids, 43, 1875-1886 (1995)

6. P.-E. Dumouchel, J.-J. Marigo, and M. Charlotte, Rupture dynamique et fissuration quasi-statique instable, Comptes Rendus Mécanique, 335, 708-713 (2007)

7. P.-E. Dumouchel, J.-J. Marigo, and M. Charlotte, Dynamic fracture: an example of convergence towards a discontinuous quasi-static solution, Contin. Mech. Thermodyn., 20, 1-19 (2008)

8. L. Evans and R. Gariepy, Measure theory and fine properties of functions. CRC Press, Boca Raton, FL (1992)

9. G. A. Francfort and J.-J. Marigo, Revisiting brittle fracture as an energy minimization problem, J. Mech. Phys. Solids, 46, 1319-1342 (1998)

10. L. B. Freund, A simple model of the double cantilever beam crack propagation specimen, J. Mech. Phys. Solids, 25, 69-79 (1977)

11. L. B. Freund, Dynamic Fracture Mechanics. Cambridge University Press, Cambridge (1998)

12. A. Griffith, The phenomena of rupture and flow in solids, Philos. Trans. R. Soc. Lond. Ser. A, CCXXI, 163-198, (1920)

13. K. Hellan, Debond dynamics of an elastic strip, I: Timoshenko-beam properties and steady motion, Int. J. Fracture, 14, 91-100 (1978)

14. K. Hellan, Debond dynamics of an elastic strip, II: Simple transient motion, Int. J. Fracture, 14, 173-184 (1978)

15. K. Hellan, An alternative one-dimensional study of dynamic crack growth in dcb test specimens, Int. J. Fracture, 17, 311-319 (1981)

16. A. Jaubert and J.-J. Marigo, Justification of Paris-type fatigue laws from cohesive forces model via a variational approach, Contin. Mech. Thermodyn., 18, 23-45 (2006)

17. B. Lawn, Fracture of Brittle Solids - Second Edition. Cambridge University press, Cambridge (1993)

18. J.-J. Marigo, Initiation of cracks in Griffith's theory: an argument of continuity in favor of global minimization, J. Nonlinear Sci., 20, 831-868 (2010)

19. A. Mielke, Evolution of rate-independent systems, in Evolutionary equations, volume II of Handb. Differ. Equ., pages 461-559. Elsevier/North-Holland, Amsterdam (2005)

20. Q. S. Nguyen, Stability and Nonlinear Solid Mechanics. Wiley \& Son, London (2000)

21. J. W. Obreimoff, The splitting strength of mica, Proc. R. Soc. Lond. Ser. A, 127, 290-297 (1930)

22. N. Sridhar, R. Massabò, B. N. Cox, and I. J. Beyerlein, Delamination dynamics in through-thickness reinforced laminates with application to dcb specimen, Int. J. Fracture, 118, 119-144 (2002)

23. C. Truesdell and R. Toupin, The Classical Field Theories, in S. Flügge, editor, Handbuch der Physik, volume III/1. Springer-Verlag, Berlin (1960) 